\title{
Stereoselektive Aromatenalkylierung mit Threonin-trifluormethansulfonaten ${ }^{1,2)}$
}

\author{
Franz Effenberger* und Thomas Weber ${ }^{3)}$ \\ Institut für Organische Chemie der Universität Stuttgart, \\ Pfaffenwaldring 55, D-7000 Stuttgart 80
}

Eingegangen am 2. September 1987

\begin{abstract}
Umsetzung der $N$-Phthaloylthreonin-oder -allothreonin-methylester 3 mit Trifluormethansulfonsäureanhydrid/Pyridin in Dichlormethan führt in quantitativer Ausbeute zu Threonin- bzw. Allothreonin-trifluormethansulfonaten 4, mit denen sich Aromaten in Gegenwart von Trifluormethansulfonsäure unter Erhaltung der Konfiguration an C-2 der Aminosäuren zu $\beta$-Methyl- $N$ phthaloylphenylalanin-methylestern 6 alkylieren lassen. Bezüglich der Konfiguration an C-3 der Aminosäuren reagieren die Threonin-Derivate $4 \mathrm{~b}(2 R, 3 S)$ und $4 \mathrm{c}(2 S, 3 R)$ unter fast vollständiger Retention $z u$ 6e $(2 R, 3 R)$ und $6 f(2 S, 3 S)$, während mit den Allothreonin-Derivaten $4 \mathrm{e}(2 R, 3 R)$ und $4 \mathrm{f}(2 S, 3 S)$ wejtgehend $\mathrm{Ra}$ cemisierung erfolgt. Die überraschende stereoselektive Aromatenalkylierung mit den Verbindungen $4 \mathrm{~b}, \mathrm{c}$ wird durch Rotationshinderung bei gleichzeitiger Rückseitenabschirmung des intermediären Carbokations durch die $\mathrm{N}$-Phthaloylgruppe gedeutet.
\end{abstract}

Synthesen natürlicher und unnatürlicher $\alpha$-Aminosäuren sind wegen der biologischen Wirksamkeit dieser Verbindungen von allgemeinem Interesse ${ }^{4)}$. In früheren Arbeiten ") haben wir über die Darstellung der Trifluormethansulfonate optisch aktiver $\alpha$-Hydroxycarbonsäureester berichtet, die sich mit Nucleophilen, wie z. B. Aminen, stereoselektiv unter Walden-Umkehr in $N$-substituierte $\alpha$-Aminosäureester überführen lassen. Überraschenderweise konnten unter Friedel-Crafts-Bedingungen auch Aromaten mit $x$ (Methylsulfonyloxy)carbonsäureestern unter Konfigurationsumkehr 7.u $\alpha$-Arylcarbonsäureestern umgesetzt werden ${ }^{6)}$. Die Übertragung dieses Reaktionstyps auf $\beta$-Hydroxycarbonsäureester sollte ausgehend von den leicht zugänglichen natürlichen Aminosäuren Serin und Threonin - in einfacher Weise zu $\beta$-Arylalaninen bzw. $\beta$-Aryl- $\beta$-methylalaninen führen.

Bei der Untersuchung dieser Reaktion mit Threonin war für uns neben der Darstellung der $\beta$-Aryl- $\beta$-methyl- $\alpha$-aminosäuren der stereochemische Verlauf der Reaktion bezüglich der Konfiguration am $\beta$-C-Atom des Threonins aus der Sicht einer Aromatenalkylierung interessant.

Im Gegensatz zu den Reaktionen $\alpha$-substituierter Carbonsäurederivate mit Nucleophilen ist bei den L'msetzungen entsprechend $\beta$-substituierter Verbindungen bevorzugt mit Eliminierung zu den energetisch begünstigten $\alpha, \beta$-ungesättigten Carbonsäurederivaten zu rechnen. Dies ist vermutlich auch der Grund dafür, daß bisher keine Alkylierungsreaktion von $\beta$-Halogen- oder $\beta$-Sulfonyloxy- $\alpha$ aminosäurederivaten mit Aromaten beschrieben worden sind.

\section{Darstellung von Threonin- und Allothreonintrifluor-} methansulfonaten

Alkyltrifluormethansulfonate sind in den letzten Jahren auf vielfältige Weise dargestellt worden ${ }^{7}$. Dabei hat sich die Veresterung von Hydroxyverbindungen mit Trifluormethansulfonsäureanhydrid (TFMSA) in Gegenwart äquimolarer Mengen Pyridin besonders bewährt ${ }^{63.81}$

\section{Stereaselective Alkylation of Arenes with Threonine Trifluoro- methanesulfonates ${ }^{1,2)}$}

$N$-Phthaloylthreonine and -allothreonine methyl esters 3 react with trifluoromethanesulfonic anhydride/pyridine in dichloromethane to give threonine and allothreonine, respectively, trifluoromethanesulfonates 4 in quantitative yields. Arenes can be alkylated with compounds 4 to yield $\beta$-methyl- $N$-phthaloylphenylalanine methyl esters 6 without any racemization at $C-2$ of the amino acids. With respect to $C-3$ of the amino acids, the threonine derivatives $4 \mathrm{~b}(2 R, 3 S)$ and $4 \mathrm{c}(2 S, 3 R)$ react to give $6 \mathrm{e}(2 R, 3 R)$ and 6f $(2 S, 3 S)$ with almost complete retention of configuration, whereas the allothreonine derivatives $4 \mathrm{e}, \mathrm{f}$ suffer from extensive racemization. The unexpected stereoselectivity of the alkylation with compounds $4 b, c$ is explained by steric hindrance of rotation and simultaneous shielding of the backside of the intermediate carbocation by the phthaloyl group.

Für Alkylierungsreaktionen mit Threonin- und Allothreonin-trifluormethansulfonaten müssen deren Amino- und Säure-Gruppen säurestabil geschützt werden. Nachdem sich die Phthaloylgruppe beim Arbeiten unter Friedel-Crafts-Bedingungen als besonders geeignet erwiesen hat ${ }^{9}$, haben wir sie auch bei diesen Reaktionen angewandt. Die $N$-Phthaloyl-geschützten racemischen $\mathbf{3 a}$ und optisch reinen Threonin-methylester 3b,c wurden mit TFMSA/Pyridin in Dichlormethan als Lösungsmittel umgesetzt. Nach beendeter Reaktion wurde Dichlormethan entfernt und durch Tetrachlorkohlenstoff ersetzt. Auf diese Weise fiel Pyridiniumtrifluormethansulfonat vollständig aus, so da $B$ die Threonintriflate $\mathbf{4 a}-\mathbf{c}$ in praktisch quantitativer Ausbeute aus den

Tab. 1. N-Phthaloyl- $O$-(trifluormethylsulfonyl)threonin-methylester $4 \mathbf{a}-\mathbf{c}$ aus den $N$-Phthaloylthreonin-methylestern $\mathbf{3 a}-\mathbf{c}$ und TFMSA/Pyridin sowie $N$-Phthaloyl- $O$-(trifluormethylsulfonyl)allothreonin-methylester $4 \mathrm{e}, \mathrm{f}$ durch Hydrolyse von $4 \mathrm{~b}, \mathrm{c} z \mathrm{zu}$ den $N$ Phthaloylallothreonin-methylestern 3e,f, deren Umsetzung mit TFMSA/Pyridin und Aufarbeitung mittels MPLC

\begin{tabular}{|c|c|c|c|c|c|c|c|c|}
\hline \multirow[b]{2}{*}{3} & \multicolumn{2}{|c|}{ Edukte } & \multirow[b]{2}{*}{$\begin{array}{c}{[\boldsymbol{\alpha}] \boldsymbol{B}^{D}} \\
\{c=1 \\
(\mathrm{CHCl})\end{array}$} & \multirow[b]{2}{*}{4} & \multirow[b]{2}{*}{$\begin{array}{c}\text { Konfigu- } \\
\text { ration }\end{array}$} & \multicolumn{2}{|c|}{ Produkte } & \multirow[b]{2}{*}{$\begin{array}{c}{[\alpha] \stackrel{p}{ }} \\
(c=1 . \\
\left.\mathrm{CCl}_{4}\right)\end{array}$} \\
\hline & $\begin{array}{l}\text { Konfigu- } \\
\text { ration }\end{array}$ & $\begin{array}{l}\text { Schmp. } \\
{[\mathrm{C}]}\end{array}$ & & & & $\begin{array}{l}\text { Ausb. } \\
(\%)\end{array}$ & $\begin{array}{c}\text { Schmp. } \\
{\left[{ }^{\circ} \mathrm{C}\right]}\end{array}$ & \\
\hline $3 \mathbf{a}$ & $\begin{array}{l}(2 R, 3 S) \\
2 S, 3 R) \\
\text { (Racemat) }\end{array}$ & 95 & - & 42 & $\begin{array}{l}(2 R, 3 S) \\
2 S, 3 R) \\
\text { (Racemal) }\end{array}$ & 99 & $O 1$ & - \\
\hline 3b & $(2 R, 3 S)$ & $96-97.5$ & -26 & $4 b$ & $(2 R, 3 S)$ & 100 & 01 & +46.7 \\
\hline 3c & $(2 S, 3 R)$ & $97-98.5$ & +26.4 & $4 c$ & $(2 S, 3 R)$ & 100 & $\mathrm{Ol}$ & -44.1 \\
\hline $3 d$ & $\begin{array}{l}(2 R, 3 R /) \\
2 S .3 S) \\
\text { (Racemat) }\end{array}$ & 01 & - & & & & & \\
\hline $3 \mathbf{e}$ & $(2 R, 3 R)$ & 01 & +9.5 & $4 e$ & $(2 R, 3 R)$ & 99 & $109-111$ & +93.4 \\
\hline $3 f$ & $(2 S .3 S)$ & $\varnothing 1$ & -9.6 & $4 f$ & $(2 S, 3 S)$ & 100 & $109-111$ & -93.1 \\
\hline
\end{tabular}


Filtraten als farblose Öle isoliert werden konnten. Die feuchtigkeitsempfindlichen Substanzen wurde jeweils sofort weiter umgesetzt.

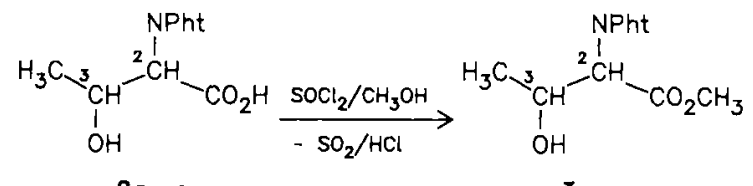

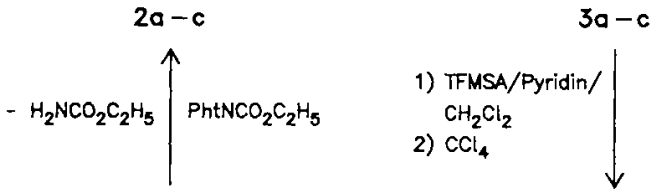

$$
\begin{aligned}
& { }_{\mathrm{OH}}^{\mathrm{H}}{ }_{3}{ }_{3}{ }^{2} \stackrel{\mathrm{NH}^{\mathrm{C}}}{\mathrm{CH}}-\mathrm{CO}_{2} \mathrm{H} \\
& \text { 1a-c }
\end{aligned}
$$

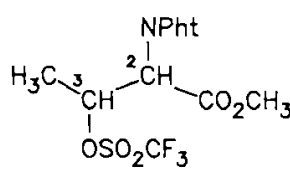

$$
\begin{aligned}
& \text { 3o, } 3
\end{aligned}
$$

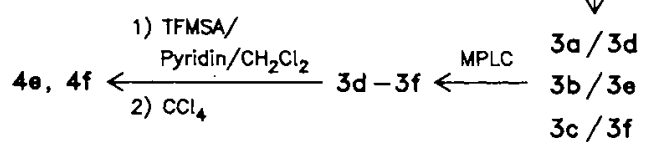

\begin{tabular}{cccccc}
\hline a & b & c & d & e & $f$ \\
\hline$(2 R / 3 S /$ & $(2 R / 3 S)$ & $(2 S, 3 R)$ & $\begin{array}{c}(2 R / 3 R / \\
2 S / 3 S)\end{array}$ & $(2 R / 3 R)$ & $(2 S / 3 S)$ \\
$2 S / 3 R)$ & & & &
\end{tabular}

Pht $=$ Phthaloyl. - TFMSA $=$ Triflu ormethansulfonsäureanhydrid.MPLC $=$ Mitteldruckchromatogrophie

Die Synthese der $N$-Phthaloylallothreonin-triflate $4 \mathrm{e}, \mathrm{f} \mathrm{er-}$ folgte analog; wobei die Produkte ebenfalls in quantitativer Ausbeute, jedoch als farblose Kristalle anfielen. Die $\mathrm{N}$ Phthaloylallothreonin-methylester $\mathbf{3 d}-\mathbf{f}$ erhielten wir durch Hydrolyse der $N$-Phthaloylthreonintriflate $4 \mathbf{a}-\mathbf{c}$ bei Raumtemperatur, wobei teilweise Racemisierung an C-3 (Diastereomerenbildung) erfolgte. Aus den dabei resultierenden Gemischen an 3a/3d, 3b/3e und 3c/3f ließen sich nach chromatographischer Reinigung über Kieselgel die optisch reinen $N$-Phthaloylallothreonin-methylester 3d-f mittels MPLC in 50 bzw. 75\% Ausb. abtrennen. Die Enantiomerenreinheit der so isolierten Verbindungen haben wir am Beispiel des (2S,3S)-Enantiomeren 3f ${ }^{1} \mathrm{H}$-NMR-spektroskopisch unter Zugabe von ca. $10 \mathrm{~mol}-\%$ Tris[3-(heptafluorpropylhydroxymethylen)- $d$-camphorato] europium(III) als Shiftreagenz nachgewiesen ${ }^{10}$. In Gegenwart von Eu(hfc) ${ }_{3}$ spaltet das Singulett der Methylester-Gruppe des Racemats 3d in ein Dublett bei tieferem Feld auf, während das Enantiomere 3f nur ein Singulett bei tieferem Feld zeigt, das anhand von 3d eindeutig zugeordnet werden kann (s. Tab. 6).

\section{Aromatenalkylierung mit Threonin- und Allothreonintriflaten 4}

Die bei der Alkylierung von Benzol mit den Triflaten $4 \mathrm{zu}$ erwartende 2-Amino-3-phenylbuttersäure wurde schon von Fischer und Schmitz ${ }^{11)}$ durch Aminierung der entsprechenden 2-Brom-Verbindung hergestellt. 1957 wurde $\beta$-Methylphenylalanin aus Hydro- lysaten von Bottromycin, einem natürlich vorkommenden Antibiotikum, isoliert ${ }^{12)}$, dessen L-erythro-Konfiguration $(2 S, 3 S)$ wurde jedoch erst 1969 über die chemische Synthese bewiesen ${ }^{13)}$.

Am Beispiel der Umsetzung von Benzol mit den Triflaten 4 haben wir zunächst die optimalen Reaktionsbedingungen für die Alkylierung ermittelt sowie ihren stereochemischen Verlauf untersucht. So konnten wir nach $10 \mathrm{~h}$ Reaktionsdauer bei $80^{\circ} \mathrm{C}$ unter Stickstoff in überschüssigem Benzol mit den Triflaten $\mathbf{4 b}, \mathbf{c}$ in Gegenwart äquimolarer Mengen TFMS und nach Reinigung mittels MPLC jeweils ein Diastereomeres des $\beta$-Methyl- $N$-phthaloylphenylalanin-methylesters 6 in hohem Diastereomerenüberschuß isolieren (Tab. 2). Dagegen erfolgte mit den Allothreonin-triflaten $4 \mathbf{e}, \mathbf{f}$ die Alkylierung von Benzol unter analogen Bedingungen nur mit geringer Stereoselektivität.

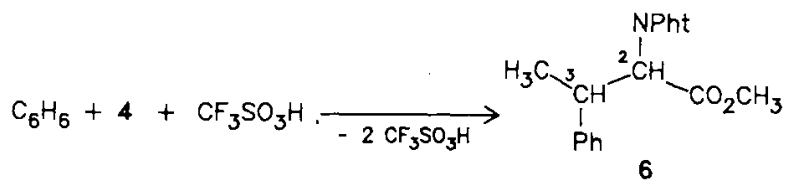

Stereochemie s. ersten Formelblock und Tab. 2

Tab. 2. $\beta$-Methyl- $N$-phthaloylphenylalanin-methylester 6 aus Benzol und $N$-Phthaloyl- $O$-(trifluormethylsulfonyl)threonin- $4 \mathbf{a}-\mathbf{c}$ bzw. -allothreonin-methylestern $\mathbf{4 e , f}$ in Gegenwart äquimolarer Mengen Trifluormethansulfonsäure (TFMS) (Reaktionszeit: $10 \mathrm{~h}$

\begin{tabular}{|c|c|c|c|c|c|c|}
\hline 4 & $\begin{array}{l}\text { Edukt } \\
\text { Konfigu- } \\
\text { ration }\end{array}$ & 6 & $\begin{array}{l}\text { Produ } \\
\text { Konfigu- } \\
\text { ration }\end{array}$ & $\begin{array}{l}\text { Ausb. } \\
(\%)\end{array}$ & $\left(c=1,{ }^{[\alpha]_{D}^{\infty}}\right.$ & $\begin{array}{l}\text { proz. } \\
\text { Ausbeute- } \\
\text { verhältnis }\end{array}$ \\
\hline $4 a$ & $\begin{array}{l}(2 R, 3 S / \\
2 S, 3 R) \\
\text { (Racemat) }\end{array}$ & 6d & $\begin{array}{l}(2 R, 3 R) \\
2 S, 3 S) \\
\text { (Racemat) }\end{array}$ & 28 & - & - \\
\hline $4 b$ & $(2 R, 3 S)$ & $\begin{array}{r}6 e \\
+6 b\end{array}$ & $\begin{array}{l}(2 R, 3 R) \\
(2 R, 3 S)\end{array}$ & $\begin{array}{c}25 \\
0.5\end{array}$ & $\begin{array}{l}+139 \\
+121\end{array}$ & $6 e: 6 b-97.9: 2.1$ \\
\hline $4 c$ & $(2 S, 3 R)$ & $\begin{array}{r}6 f \\
+6 c\end{array}$ & $\begin{array}{l}(2 S, 3 S) \\
(2 S, 3 R)\end{array}$ & $\begin{array}{c}23 \\
0.8\end{array}$ & $\begin{array}{l}-140 \\
-120\end{array}$ & $6 f: 6 c=96.7: 3.3$ \\
\hline $4 e$ & $(2 R, 3 R)$ & $\begin{array}{r}6 e \\
+6 b\end{array}$ & & $\begin{array}{l}6.7 \\
4.4\end{array}$ & $\begin{array}{l}+139(c=1.5) \\
+120(c=0.5)\end{array}$ & $6 \mathrm{e}: 6 \mathrm{~b}=60,0: 40,0$ \\
\hline $4 f$ & $(2 S, 3 S)$ & $\begin{array}{r}6 f \\
+\quad 6 c\end{array}$ & & $\begin{array}{l}8.8 \\
6.0\end{array}$ & $\begin{array}{l}-139 \\
-120.5(c=0.6)\end{array}$ & $6 f: 6 c=59.3: 40.7$ \\
\hline
\end{tabular}
bei $80^{\circ} \mathrm{C}$ )

a) Die ca. $3 \%$ Anteile an $(2 R, 3 S)$ und $(2 S, 3 R)$ wurden vernachlässigt.

Die bei der Reaktion frei werdende TFMS läßt sich praktisch quantitativ als Bariumsalz zurückgewinnen, aus dem durch Erhitzen mit 100proz. Schwefelsäure i. Vak. dic TFMS abdestilliert werden $k^{2}{ }^{14}$ ).

Die Enantiomerenreinheit der erhaltenen Produkte 6 haben wir wiederum ${ }^{1} \mathrm{H}$-NMR-spektroskopisch mit dem Shiftreagenz $\mathrm{Eu}(\mathrm{hfc})_{3}$ am Beispiel von $6 \mathrm{~d}(2 R, 3 R / 2 S, 3 S)$ nachgewiesen (s. Tab. 6). Zur Bestimmung der Relativkonfiguration der erhaltenen Diastereomeren haben wir einmal die vicinalen Kopplungskonstanten der $\mathrm{CHCH}_{3}$ - und $\mathrm{CHN}$ Protonen herangezogen, die im Falle der erythro-Konfiguration größer sein müßten als im Falle der threo-Konfiguration $^{13.15)}$. Da wir für diese Kopplungskonstanten für das Enantiomerenpaar 6e $(2 R, 3 R), 6 \mathrm{f}(2 S, 3 S) J=10.8 \mathrm{~Hz}$ und für das Enantiomerenpaar $6 \mathbf{b}(2 R, 3 S), 6 \mathbf{c}(2 S, 3 R) J=9.9 \mathrm{~Hz}$ gefunden haben (s. exp. Teil), ist für $\mathbf{6 e}, \mathbf{f}$ die erythro- und für $6 \mathbf{b}, \mathbf{c}$ die threo-Konfiguration wahrscheinlich. Zum an- 
deren haben wir den bei der Alkylierung mit 4c $(2 S, 3 R)$ erhaltenen $\beta$-Methyl- $N$-phthaloyl-(2S,3S)-phenylalanin-methylester $(6 \mathrm{f})$ in das freie $\beta$-Methyl-(2S,3S)-phenylalanin (7) übergeführt und dieses anschließend zum $N$-Acetyl- $\beta$-methyl-(2S,3S)-phenylalanin (8) acyliert; letzteres stimmt mit dem in der Literatur ${ }^{16}$ beschriebenen Produkt überein. Wie bereits oben angeführt, gilt die erythro-Konfiguration des in Bottromycin enthaltenen $\beta$-Methyl- $(2 S, 3 S)$-phenylalanins (7) als gesichert ${ }^{13.16}$. Damit liegt ein weiterer Beweis für die erythro-Konfiguration von $\mathbf{6 f}$ vor.

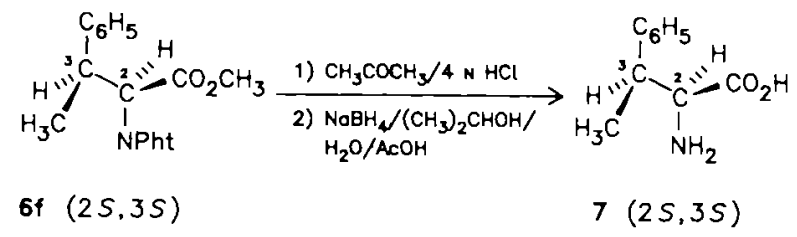

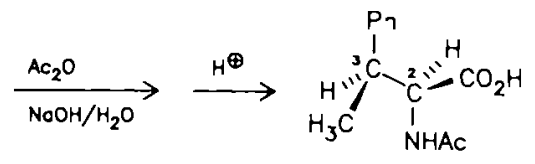

$$
\begin{aligned}
& 8(2 S, 3 S)
\end{aligned}
$$

Die endgültige Bestätigung der Absolutkonfiguration von $6 f(2 S, 3 S)$ haben wir über eine Röntgenstrukturanalyse ${ }^{17)}$ erhalten; die Torsionswinkel (Tab. 3) beweisen eindeutig die erythro-Konfiguration ${ }^{18)}$. Demnach müssen die Enantiomeren des Diastereomerenpaars $6 \mathrm{~b}(2 R, 3 S), 6 \mathrm{c}(2 S, 3 R)$ in der threo-Konfiguration vorliegen.

Tab. 3. Torsionswinkel [Atom(1)$\operatorname{Atom}(2)-\operatorname{Atom}(3)-\operatorname{Atom}(4)]$ in $\beta-\mathrm{Me}$ thyl- $N$-phthaloyl- $(2 S, 3 S)$-phenylalanin-methylester $(6 \mathrm{f})$

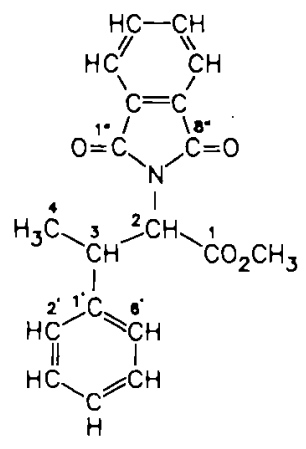

\begin{tabular}{lllll}
\hline Atom (1) & Atom (2) & Atom (3) & Atom (4) & $\begin{array}{r}\text { Torsions- } \\
\text { winkel [”] }\end{array}$ \\
\hline N & C-2 & C-3 & C-1' & -41.2 \\
N & C-2 & C-3 & C-4 & -162.4 \\
C-1 & C-2 & C-3 & C-4 & +72.2 \\
C-1 & C-2 & C-3 & C-1' & -166.7 \\
C-2 & C-3 & C-1' & C-2' & -61.5 \\
C-2 & C-3 & C-1' & C-6' & +122.3 \\
C-4 & C-3 & C-1' & C-2' & +59.9 \\
C-4 & C-3 & C-1' & C-6' & -116.8 \\
C-1" & N & C-2 & C-1 & +71.2 \\
C-1" & N & C-2 & C-3 & -56.8 \\
C-8" & N & C-2 & C-1 & -111.5 \\
C-8" & N & C-2 & C-3 & +120.4 \\
\hline
\end{tabular}

Neben der Alkylierung von Benzol mit Threonin-triflaten haben wir noch die von Mesitylen, Resorcin-. Brenzcate- chin- und Hydrochinon-dimethylether sowie von Indol untersucht, erhielten jedoch nur im Falle von Mesitylen und Hydrochinon-dimethylether Alkylierungsprodukte. Auch in diesen Fällen entstand mit dem Threonintriflat 4c $(2 S, 3 R)$ jeweils nur ein Enantiomeres, nämlich $\beta$-Methyl- $N$-phthaloyl-(2S,3S)-mesitylalanin-methylester $\left(6^{\prime} f\right)$ in $63 \%$ Ausb. bzw. der $\beta$-Methyl- $N$-phthaloyl- $\beta$-(2,5-dimethoxyphenyl)$(2 S, 3 S)$-alanin-methylester ( 6 " $f$ ) in $37 \%$ Ausbeute.

Offensichtlich wird eine Alkylierung der Resorcin- und Brenzcatechin-dimethylether unter den Reaktionsbedingungen (TFMS) durch die begünstigte C-Protonierung ${ }^{19)}$ erschwert. Es ist jedoch auch möglich, daß die Alkylierung in diesen Fällen schon reversibel ist ${ }^{20}$.<smiles>Cc1cc(C)cc(C)c1</smiles><smiles>COC(=O)C(=Cc1c(C)cc(C)cc1C)C(C)C</smiles><smiles>COc1ccc(OC)c(C#CC#CC2CC2)c1</smiles><smiles>COc1ccc(OC)c(C(C)C(C(C)=O)C(=O)OCc2ccccc2)c1</smiles>

Im 'H-NMR-Spektrum von 6'f erscheinen die Mesityl- $\mathrm{CH}_{3}$-Protonen-Signale in 2'- und 6'-Position bei unterschiedlichen $\delta$-Werten bedingt durch die gehinderte Rotation des Arylsubstituenten um $\mathrm{C}-1^{\prime}-\mathrm{C}-3$. Aus der Koaleszenztemperatur, bei der die C-2'- und C-6'-Methyl-Singuletts zusammenfallen, ergibt sich die freie Aktivierungsenthalpie für die Rotation um die C-1'-C-3-Bindung zu $\Delta G^{*}=82 \mathrm{~kJ} / \mathrm{mol}$.

$\mathrm{Da}$ Indol in Gegenwart von Mineralsäuren protoniert wird und polymerisiert ${ }^{21 !}$, haben wir die Alkylierung von Indol ohne Zusatz von TFMS bei Raumtemperatur bis $100^{\circ} \mathrm{C}$ mit und ohne 1,2-Dichlorethan als Lösungsmittel versucht und - um eine $N$-Alkylierung auszuschließen - das $N$-(Phenylsulfonyl)-substituierte Indol eingesetzt. Außerdem haben wir zum Abfangen der bei der Reaktion entstehenden TFMS die sterisch gehinderte Base 2,6-Di-tert-butyl4-methylpyridin zugesetzt. Wir konnten jedoch in keinem Fall ein Alkylierungsprodukt isolieren oder 'H-NMR-spektroskopisch nachweisen.

Zur präparativen Darstellung der $\beta$-Methyl- $\beta$-arylalanine 6 suchten wir nach einer vereinfachten Methode mit höheren Ausbeuten. Nachdem die Triflate $\mathbf{4}$ aus den $N$-Phthaloylthreonin-methylestern 3 mit TFMSA dargestellt werden und bei der Aromatenalkylierung mit 4 noch äquimolare Mengen an TFMS zugesetzt werden müssen, haben wir $\mathbf{3 c}$ $(2 S, 3 R)$ mit der äquimolaren Menge TFMSA und Benzol bei $80^{\circ} \mathrm{C}$ erwärmt und die Produktbildung ${ }^{1} \mathrm{H}-\mathrm{NMR}$-spek- 
troskopisch verfolgt. Auf diese Weise erhielten wir direkt ohne Isolierung des Triflats $\mathbf{4 c}$ das erwünschte Alkylierungsprodukt $6 \mathrm{f}(2 S, 3 S)$, wobei nach $10 \mathrm{~h}$ die maximale Ausbeute erreicht wird. Aus dem intermediär gebildeten Triflat $4 c$ entstanden daneben noch die Eliminierungsprodukte 9 und $\mathbf{1 0}$.

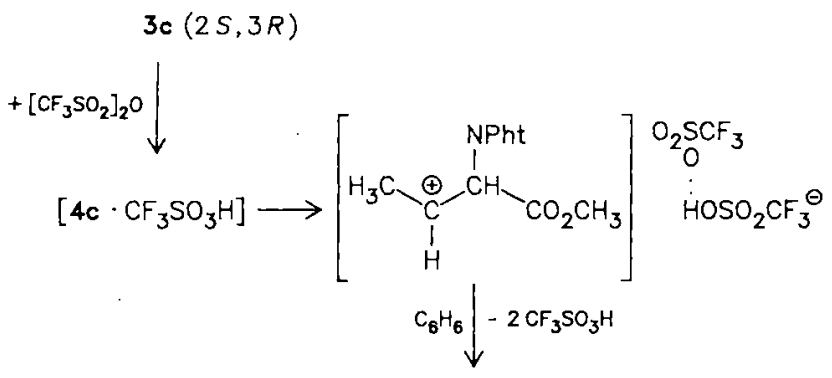

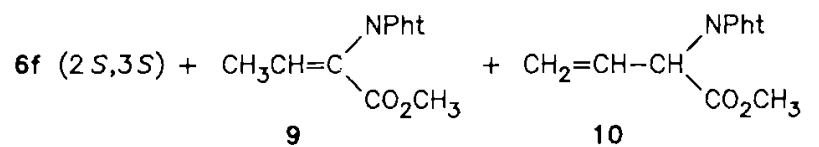

Tab. 4. Prozentuale Produktverteilung bei der Umsetzung von $\mathrm{N}$ Phthaloyl-( $2 S, 3 R)$-threonin-methylester (3c) mit TFMSA in Benzol bei $80^{\circ} \mathrm{C}\left({ }^{1} \mathrm{H}\right.$-NMR-spektroskopisch bestimmt)

\begin{tabular}{|c|c|c|c|c|}
\hline $\begin{array}{l}\text { Zeit } \\
{[\mathrm{h}]}\end{array}$ & $\begin{array}{c}\text { B-Methyl- } \\
N \text {-phthaloyl- } \\
(2 S, 3 S)- \\
\text { phenylalanin- } \\
\text { methylester } \\
\text { (6f) }\end{array}$ & $\begin{array}{c}N \text {-Phthaloyl- } \\
\text { 2,3-didehydro- } \\
\text { alanin- } \\
\text { methylester } \\
\text { (9) }\end{array}$ & $\begin{array}{l}N \text {-Phthaloyl- } \\
\text { vinylglycin- } \\
\text { methylester } \\
(\mathbf{1 0})\end{array}$ & $\begin{array}{l}\text { Nicht } \\
\text { umge- } \\
\text { setztes } \\
\text { Edukt } \\
\mathbf{3 c}\end{array}$ \\
\hline $\begin{array}{r}2 \\
4 \\
6 \\
8 \\
10\end{array}$ & $\begin{array}{r}7.7 \\
16.7 \\
25.9 \\
31.9 \\
35.0\end{array}$ & $\begin{array}{r}6.6 \\
13.3 \\
17.0 \\
20.0 \\
22.7\end{array}$ & $\begin{array}{l}17.3 \\
26.1 \\
32.8 \\
33.0 \\
33.0\end{array}$ & $\begin{array}{r}68.4 \\
43.9 \\
24.3 \\
13.4 \\
9.5\end{array}$ \\
\hline
\end{tabular}

Bei längerem Erhitzen erfolgte keine Ausbeutesteigerung mehr an 6f, lediglich in zunehmendem Maße Zersetzung der Verbindungen 9 und 10. In einem präparativen Ansatz konnte unter diesen Bedingungen $6 \mathrm{f}$ in $23 \%$ Ausbeute erhalten werden. Der Vorteil dieser „Eintopfmethode“ besteht nicht nur in der vereinfachten Durchführung der Reaktion, sondern auch in der Erzielung einer noch höheren optischen Ausbeute; im vorliegenden Fall konnte neben $6 f$ überhaupt kein 6c $(2 S, 3 R)$ mehr nachgewiesen werden. Da sich bei der Eintopfmethode das eingesetzte TFMSA praktisch quantitativ als TFMS rückisolieren läßt, stellt dieses Verfahren eine gute präparative Methode zur Darstellung optisch reiner $\beta$-Methyl- $\beta$-arylalanine dar.

Versuche, die Alkylierung von Benzol mit 4 durch $\mathrm{AlCl}_{3}$ zu katalysieren, ergaben deutlich schlechtere Ausbeuten an 6 bei Abnahme der Stereoselektivität der Reaktion an C-3 und zusätzlicher Racemisierung an C-2 (s. Tab. 7, exp. Teil). Ersetzt man die Trifluormethansulfonat-Austrittsgruppe in 4 durch Mesylat, so erfolgt die Alkylierung von Benzol deutlich schlechter als mit dem Triflat, während mit $\beta$-Chlor- $\beta$ methyl- $N$-phthaloyl- $(2 S, 3 R)$-alanin-methylester unter Friedel-Crafts-Bedingungen mit überschüssigen Mengen an $\mathrm{AlCl}_{3}$ oder $\mathrm{FeCl}_{3}$ überhaupt keine Alkylierung mehr erzielt werden konnte.

\section{Deutung des stereochemischen Ablaufs der Aromatenalkylierung mit $N$-Phthaloylthreonin- triflaten 4}

Den stereochemischen Ablauf der Aromatenalkylierung haben wir am Beispiel der Alkylierung von Benzol untersucht (siehe oben), bei den anderen mit Erfolg umgesetzten Aromaten Mesitylen und Hydrochinon-dimethylether sind jedoch die stereochemischen Befunde vergleichbar.

Wie bereits erwähnt, verlaufen die Aromatenalkylierungen mit den Triflaten 4 unter den angewandten Bedingungen in allen Fällen unter Erhaltung der Konfiguration an C-2 der Aminosäureester 4. Schwierig vorauszusagen war die Stereochemie an C-3 der bei der Alkylierung gebildeten $\beta$ Methylphenylalanin-Derivate 6, da bei der geringen $\mathrm{Nu}-$ cleophilie des Benzols eine $S_{N} 1$-Typ-Alkylierung mit Carbokationen als Reaktionszwischenstufen angenommen werden muß.

Die Friedel-Crafts-Alkylierung von Aromaten mit optisch aktiven Alkylierungsmitteln erfolgt im allgemeinen unter weitgehender Racemisierung ${ }^{22}$. Insbesondere Suga und Mitarb. haben jedoch gezeigt, daß Alkylierungen mit optisch aktiven Verbindungen auch weitgehend racemisierungsfrei verlaufen können, wenn bestimmte strukturelle Voraussetzungen gegeben sind ${ }^{6 a, 23 i}$. In der Regel kommt es bei diesen Alkylierungen zur Inversion der Konfiguration am angreifenden C-Atom des Alkylierungsagens. Retention wurde bei der Alkylierung mit $\beta$-Phenylalkylhalogeniden gefunden, wobei zuerst durch intramolekulare Alkylierung eine Cyclohexadienylium-Zwischenstufe entsteht, die in einem zweiten Schritt mit dem Aromaten reagiert; so resultiert durch zweimalige Inversion eine Retention ${ }^{24}$. Alle bekannten experimentellen Befunde besagen, daB bei nucleophilen Substitutionen an Chiralitätszentren, die über Carbokationen als Reaktionszwischenstufen ablaufen, nur dann keine Racemisierung erfolgt, wenn durch Nachbargruppen eine einseitige Abschirmung des Reaktionszentrums gegeben ist.

Bei der Dissoziation der Triflate $4 \mathrm{kann}$ sowohl die $\mathrm{N}$ Phthaloyl(NPht)-als auch die Ester-Gruppe (E) als Nachbargruppe wirken; bei den Threonin-Derivaten $\mathbf{4 b}, \mathbf{c}$ müßten dann je nach beteiligter Nachbargruppe aus den Konformationen $\mathbf{A}$ bzw. B die Carbokationen $\mathbf{A}^{\prime}$ bzw. $\mathbf{B}^{\prime}$, bei den Allothreonin-Derivaten $\mathbf{4 e}, \mathbf{f}$ aus den Konformationen $\mathbf{C}$ bzw. D die Carbokationen $\mathbf{C}^{\prime}$ bzw. $\mathbf{D}^{\prime}$ entstehen.

Aus sterischen Gründen sollten $\mathbf{B}$ und $\mathbf{C}$ sowie $\mathbf{B}^{\prime}$ und $\mathbf{C}^{\prime}$ begünstigt sein. Aus den bei der Alkylierung von Benzol mit den Threonin-Derivaten $\mathbf{4 b}$, c mit hoher Diastereomerenausbeute entstehenden Alkylierungsprodukten 6e,f (Tab. 2) muß man jedoch schließen, daß $\mathbf{4 b}$, $\mathbf{c}$ aus der Konformation A reagieren, d. h. daß der $N$-Phthaloyl-Rest und nicht die Estergruppe die Nachbargruppenbeteiligung eingeht. Im Carbokation $\mathbf{A}^{\prime}$ wird die Rotation um die zentrale C2 -- C3Bindung offensichtlich durch die Stellung der Methylgruppe zwischen der sperrigen $N$-Phthaloyl- und Estergruppe erschwert und damit verlangsamt. Da außerdem eine einseitige Abschirmung des Kations durch den $N$-Phthaloyl-Rest erfolgt, wird die Reaktion des Benzols mit dem konfigurationsstabilen Kation von der Unterseite her erfolgen, was die große Stereoselektivität dieser Alkylierung erklärt.

Nimmt man für die Dissoziation der Allothreonin-Derivate $4 \mathrm{e}, \mathrm{f}$ ebenfalls eine Nachbargruppenbeteiligung durch den $N$-Phthaloyl-Rest an, so müßte in dem aus dem Kon- 


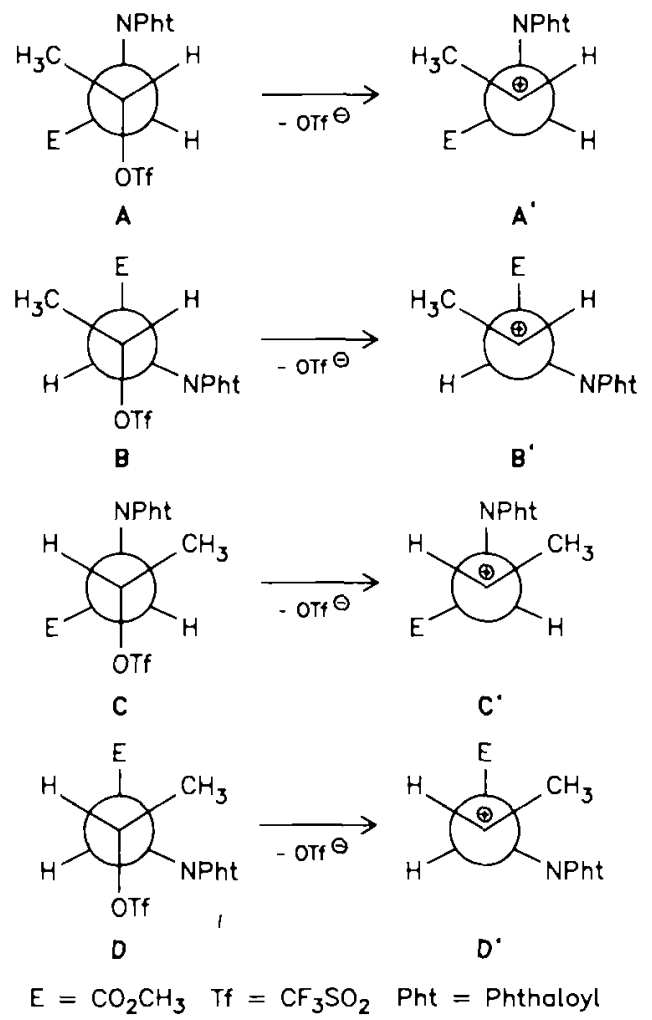

formeren $\mathbf{C}$ entstehenden Carbokation $\mathbf{C}^{\prime}$ die Rotation um $\mathrm{C} 2-\mathrm{C} 3$ jedoch sterisch weniger gehindert sein, was die geringe Stereoselektivität bei der Aromatenalkylierung mit $\mathbf{4 e , f}$ verständlich macht. Es überwiegen sogar in diesen Fällen die Diastereomeren, die sich aus der Reaktion der konformeren Carbokationen $\mathbf{A}^{\prime}$ ableiten.

Daß bei den diskutierten Reaktionen der $N$-PhthaloylRest und nicht die Estergruppe die Nachbargruppenbeteiligung eingeht, haben wir durch die Alkylierung von Benzol einerseits mit den 3-(Trifluormethylsulfonyloxy)buttersäuremethylestern 12 und andererseits den 1-Phthalimido-2-(trifluormethylsulfonyloxy)propanen 16 zusätzlich gesichert.

Bei den Umsetzungen der aus den $(R)$ - bzw. (S)-3-Hydroxybuttersäure-methylestern 11 dargestellten Triflate $(R)-12$ und (S)-12 mit Benzol erhielten wir racemischen 3-Phenylbuttersäure-methylester (13) in großen Ausbeuten.

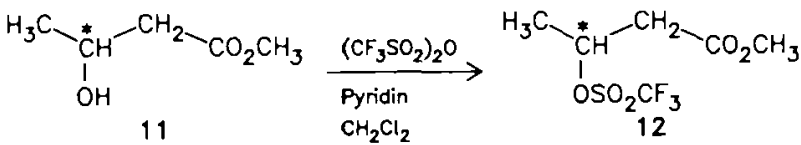

$$
\begin{aligned}
& \left.\mathrm{CF}_{3} \mathrm{SO}_{3} \mathrm{H}\right\rfloor \mathrm{C}_{8} \mathrm{H}_{6} \\
& \underset{\substack{\mathrm{CH}_{3} \\
\mathrm{C}_{6} \mathrm{H}_{5}}}{-\mathrm{CH}_{2}-\mathrm{CO}_{2} \mathrm{CH}_{3}}
\end{aligned}
$$

Dagegen erfolgte die Alkylierung von Benzol mit den Triflaten $(R)$ - und $(S)$-16 - dargestellt aus den käufliche $(R)$ und (S)-1-Amino-2-propanolen 14 über deren $N$-Phthaloylgeschützte Derivate 15 - unter teilweiser Retention der Konfiguration an $\mathrm{C}-2$ und Bildung der Enantiomerenge- mische $(R / S)$ - und $(S / R)-17$. So erhielten wir aus den Triflaten $(R)$ - bzw. (S)-16 und Benzol unter den vorstehenden Alkylierungsbedingungen $\left(10 \mathrm{~h}\right.$ bei $80^{\circ} \mathrm{C}$ in Gegenwart von TFMS) die Enantiomerengemsiche $(S / R)$ - bzw. $(R / S)-17$, deren hydrierende $N$-Phthaloyl-Schutzgruppen-Abspaltung ${ }^{25}$ ohne Veränderung der Stereochemie zu den enantiomeren $\beta$-Methylphenethylaminen 18 führt. Die optischen Ausbeuten der resultierenden Enantiomerengemische $(S / R)$ bzw. $(R / S)-18$ wurden mit Hilfe des bekannten Drehwertes für optisch reines $(R)-18$ berechnet (s. Tab. 5). Unter Berücksichtigung der nur 95-95.4proz. Reinheit der eingesetzten 1-Amino-2-propanole $(R)$ - und $(S)$-14 verliefen die Gesamtreaktionen von $(R)-14$ zu $(S / R)-18$ bzw. von $(S)-14$ $\mathrm{zu}(R / S)-18$ mit einem e.e.-Wert von $38.3-38.8 \%$.

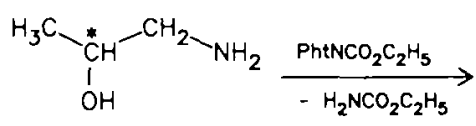

(R) -14

(S) -14

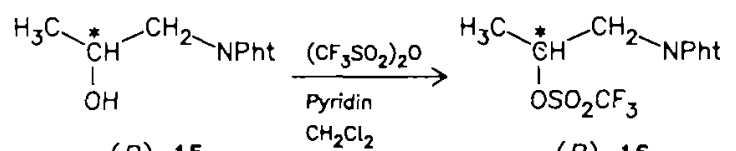

(R) -15 $\quad \mathrm{CH}_{2} \mathrm{Cl}_{2} \quad(R)-16$

(S) -15

(S) -16

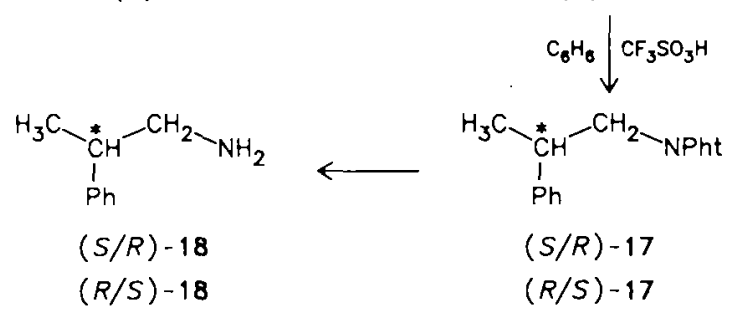

Tab. 5. Alkylierung von Benzol mit $(R)$ - und $(S)$-1-Phthalimido-2(trifluormethylsulfonyloxy)propanen $16 \mathrm{zu}(S / R)$ - und $(R / S)$ - $\beta$-Methyl- $N$-phthaloylphenethylaminen 17 sowie deren Spaltung zu

\begin{tabular}{|c|c|c|c|c|c|c|c|c|c|}
\hline \multirow[b]{2}{*}{16} & \multicolumn{2}{|c|}{ Reaktions- } & & \multirow{2}{*}{$\begin{array}{c}17 \\
\text { Ausb. } \\
(\%)\end{array}$} & \multirow[b]{2}{*}{$(R):(S)$} & \multirow{2}{*}{\multicolumn{2}{|c|}{$\begin{array}{c}18 \\
\text { Ausb. } \\
(\%)\end{array}$}} & \multirow[b]{2}{*}{$(R):(S)$} & \multirow{2}{*}{$\begin{array}{l}14 \rightarrow+18 \\
\text { e.e. }(\%)^{\prime}\end{array}$} \\
\hline & $\begin{array}{l}\text { Temp. } \\
{\left[{ }^{\circ} \mathrm{C}\right]}\end{array}$ & $\begin{array}{l}\text { Zeit } \\
{[\mathrm{h}]}\end{array}$ & & & & & & & \\
\hline & 80 & 10 & $(S / R)-17$ & 54 & c & $(S / R \vdash 18$ & 29 & $31.8: 68.2$ & 38.3 \\
\hline & 80 & 10 & $(R / S)-17$ & 55 & d) & $(R / S)-18$ & 28 & $68.5: 31.5$ & 38.8 \\
\hline (S)- & 40 & 40 & (R/S $/ 17$ & 45 & $78: 22$ & Nicht du & rchgefüh & & $58.6^{\circ)}$ \\
\hline
\end{tabular}
$(S / R)$ - und $(R / S)-\beta$-Methylphenethylaminen 18

2.b) Unter Berücksichtigung von e.e. für $(R)-14 \mathrm{a}=95 \%$ und für $(S)$ $14 \mathrm{a}=95.3 \%$. $-{ }^{b)}$ e.e. für $(S)-14 \rightarrow(R / S)-17 .{ }^{c 1}$ Analog $(S / R)-$ 18. - d) Analog $(R / S)-18$.

$\mathrm{Da} B$ die optischen Ausbeuten bei der Alkylierung erwartungsgemä $B$ von den Reaktionsbedingungen abhängig sind, zeigte die Umsetzung von Benzol mit dem Triflat (S)-16 unter milderen Alkylierungsbedingungen $\left(40 \mathrm{~h}\right.$ bei $40^{\circ} \mathrm{C}$ in Gegenwart von TFMS). Das erhaltene Enantiomerengemisch $(R / S)$-17 zeigte eine optische Ausbeute von $(R):(S)=$ 78:22 gegenüber der von 68.5:31.5 bei der Alkylierung bei $80^{\circ} \mathrm{C}$; die Gesamtreaktion von $(S)-14$ zu $(R / S)$-17 verlief bei der Alkylierung bei $40{ }^{\circ} \mathrm{C}$ mit einem e.e.-Wert von $58.6 \%$. 
Wir danken dem Fonds der Chemischen Industrie sowie der Deutschen Forschungsgemeinschaft für die Lnterstützung dieser Arbeit. Th. W. dankt dem Fonds der Chemischen Industrie für ein Doktorandenstipendium.

\section{Experimenteller Teil}

Präparative Säulenchromatographie: Säulen verschiedener Dimensionen mit Kieselgel A 60, Korngröße 0.032-0.063 mm (Fa. Riedel-de Haën). - Präparative Mitteldruck-Säulenchromatographie (MPLC): Nach Glatz ${ }^{26)}$ entwickeltes System mit Kieselgelsäulen a) $25 \times 2.4 \mathrm{~cm}$ und b) $40 \times 4.5 \mathrm{~cm}$, gefüllt mit Kieselgel (Fa. Merck) $0.015-0.025 \mathrm{~mm}$, a) 6300 und b) 9400 theoretische Böden. Detektor: UV-Detektor $(254 \mathrm{~nm})$. Laufmittel Petrolether/Ethylacetat (PE/EE). - NMR-Spektren: ${ }^{1} \mathrm{H}$ : Geräte Varian 'T $60(60$ $\mathrm{MHz})$ und EM $360(60 \mathrm{MHz})$, Bruker WP $80(80 \mathrm{MHz})$ und CXP $300(300 \mathrm{MHz}) ; \delta(\mathrm{ppm})$ bezogen auf TMS als int. Standard. - El-Massen-Spektren: Gerät MAT 711, Varian. - Drehwerte: Perkin-Elmer-Polarimeter 241 mit thermostatisierter Glasküvette $(l=1 \mathrm{dm})$.

$N$-Phthaloylthreonine 2a--c: Die käuflichen Threonine 1a-c (Degussa, Fluka, EGA) wurden nach Lit. ${ }^{27)} \mathrm{zu}$ den $N$-Phthaloylthreoninen 2a-c umgesetzt. - 2a (Racemat): Aus $13.10 \mathrm{~g}(0.11$ mol) Threonin (1 a), $83 \mathrm{ml}$ Wasser, $12.0 \mathrm{~g}(0.11 \mathrm{~mol})$ Natriumcarbonat und $24.1 \mathrm{~g}(0.11 \mathrm{~mol}) \mathrm{N}$-(Ethoxycarbonyl)phthalimid nach Aufarbeiten mit $6 \mathrm{~N} \mathrm{HCl}$; Ausb. $22.0 \mathrm{~g}(80 \%)$, Schmp. $116-118^{\circ} \mathrm{C}$ [Lit. ${ }^{28)} 119-121^{\circ} \mathrm{C}, 122-123^{\circ} \mathrm{C}$ (EE/PE)]. - 2b (2R,3S): Aus $10.0 \mathrm{~g}(0.084 \mathrm{mmol})(2 R, 3 S)$-Threonin $(\mathbf{1} \mathbf{b})$ analog $2 \mathrm{a}$; Ausb. $18.4 \mathrm{~g}$ $(88 \%)$, Schmp. $142^{\circ} \mathrm{C},[\alpha]_{\mathrm{D}}=+38.4(\mathrm{c}=1.1$, EtOH $) .-2 \mathrm{c}$ $(2 S, 3 R)$ : Aus $11.9 \mathrm{~g}(0.10 \mathrm{~mol})(2 S, 3 R)-T h r e o n i n(1 \mathrm{c})$ analog $2 \mathbf{a}$; Ausb. $22.5 \mathrm{~g}(90 \%)$, Schmp. $141-143^{\circ} \mathrm{C}$ (Lit. ${ }^{29)} 143-144^{\circ} \mathrm{C}$ ), $[\alpha]_{\mathrm{D}}^{20}=-38.5(c=3.22, \mathrm{EtOH})\left\langle\mathrm{Lit}^{29)}[\alpha]_{\mathrm{D}}^{20}=-36.7(0.048 \mathrm{~g}\right.$ in $1.5 \mathrm{ml} \mathrm{EtOH})\rangle$.

$N$-Phthaloylthreonin-methylester $\mathbf{3 a - c :}$ Aus $\mathbf{2 a - c}$ nach Lit. ${ }^{30)}$. - 3a (Racemat): Aus $22.1 \mathrm{~g}(88.5 \mathrm{mmol}) \mathbf{2 a}, 11.6 \mathrm{~g}(98.0$ mmol) Thionylchlorid und $80 \mathrm{ml}$ Methanol; Ausb. $9.5 \mathrm{~g} \mathrm{(41 \% ),}$ Schmp. $95^{\circ} \mathrm{C}$ ( Lit. $\left.^{31)} 95^{\circ} \mathrm{C}\right)$ - $3 \mathrm{~b}(2 R, 3 S)$ : Aus $18.4 \mathrm{~g}(74.0 \mathrm{mmol})$ 2b und $15.0 \mathrm{~g}$ (126.0 mmol) Thionylchlorid in $50 \mathrm{ml}$ Methanol; Ausb. $9.1 \mathrm{~g}(47 \%)$, Schmp. $96-97^{\circ} \mathrm{C},[\alpha]_{\mathrm{D}}^{20}=-26(c=1$, $\left.\mathrm{CHCl}_{3}\right)$. $-3 \mathrm{c}(2 \mathrm{~S}, 3 \mathrm{R})$ : Aus $11.0 \mathrm{~g}(44.0 \mathrm{mmol}) 2 \mathrm{c}$ und $8.0 \mathrm{~g}(67.0$ mmol) Thionylchlorid in $50 \mathrm{ml}$ Methanol; Ausb. $7.0 \mathrm{~g}(60 \%)$, Schmp. $97-98.5^{\circ} \mathrm{C},[\alpha]_{\mathrm{D}}^{20}=+26.4\left(c=1, \mathrm{CHCl}_{3}\right) .-{ }^{i} \mathrm{H}-\mathrm{NMR}$ $\left(\mathrm{CDCl}_{3}\right): \delta=1.21\left(\mathrm{~d},{ }^{3} \mathrm{~J}=6.6 \mathrm{~Hz}, 3 \mathrm{H}, \mathrm{CHCH}_{3}\right), 3.79(\mathrm{~s}, 3 \mathrm{H}$, $\left.\mathrm{COCH}_{3}\right), 4.05\left(\mathrm{~d},{ }^{3} \mathrm{~J}=9.6 \mathrm{~Hz}, 1 \mathrm{H}, \mathrm{OH}\right), 4.65\left(\mathrm{dq},{ }^{3} \mathrm{~J}=6.6 \mathrm{~Hz}\right.$, $\left.{ }^{3} J=4.3 \mathrm{~Hz}, 1 \mathrm{H}, \mathrm{CHCH}_{3}\right), 4.98\left(\mathrm{~d},{ }^{3} J=4.3 \mathrm{~Hz}, 1 \mathrm{H}, \mathrm{CHN}\right)$, $7.76-7.94(\mathrm{~m}, 4 \mathrm{H}$, Phthaloyl).

$$
\begin{array}{lllll}
\mathrm{C}_{13} \mathrm{H}_{13} \mathrm{NO}_{5} \text { (263.2) } & \text { Ber. } \mathrm{C} 59.31 \mathrm{H} 4.98 \text { N } 5.32 \\
& \text { Gef. C } 59.48 \text { H } 5.13 \text { N } 5.16
\end{array}
$$

$N$-Phthaloyl-O-(trifluormethylsulfonyl) threonin-methylester 4a-c: In einem unter Vakuum mit fächelnder Flamme ausgeheizten und mit trockenem Stickstoff belüfteten Rundkolben mit aufgesetztem Tropftrichter wird zu Trifluormethansulfonsäureanhydrid (TFMSA) in Dichlormethan der $N$-Phthaloylthreonin-methylester 3 - gemeinsam mit Pyridin in Dichlormethan gelöst langsam bei $0^{\circ} \mathrm{C}$ unter Rühren zugetropft. Das Gemisch wird $2 \mathrm{~h}$ bei $40^{\circ} \mathrm{C}$ unter trockenem Stickstoff gerührt, das Dichlormethan i. Vak. unter Feuchtigkeitsausschlu $B$ abdestilliert, der Rückstand unter leichtem Erwärmen mit $50 \mathrm{ml}$ Tetrachlorkohlenstoff versetzt und die gebildete Suspension über eine Schutzgasfritte abfiltriert. Aus dem Filtrat wird Tetrachlorkohlenstoff i. Vak. - zuletzt i. Hochvak. - abdestilliert, das zurückbleibende farblose hochviskose $\circlearrowright 1$ wird sofort weiter umgesetzt. 4a (Racemat): Aus $0.85 \mathrm{~g}$ (3.0 mmol) TFMSA in $35 \mathrm{ml}$ Dichlormethan und $0.53 \mathrm{~g}$ (2.0 mmol) 3a in $0.24 \mathrm{~g}$ (3.0 mmol) Pyridin und $15 \mathrm{ml}$ Dichlormethan; nach Aufarbeiten Ausb. $0.783 \mathrm{~g}(99 \%)$. ${ }^{1} \mathrm{H}$-NMR $\left(\mathrm{CDCl}_{3}\right): \delta=1.78\left(\mathrm{~d},{ }^{3} \mathrm{~J}=6.3 \mathrm{~Hz}, 3 \mathrm{H}, \mathrm{CHCH}_{3}\right), 3.78$ (s, $\left.3 \mathrm{H}, \mathrm{COCH}_{3}\right), 4.99\left(\mathrm{~d},{ }^{3} \mathrm{~J}=7.8 \mathrm{~Hz}, 1 \mathrm{H}, \mathrm{CHN}\right), 5.75\left(\mathrm{qd},{ }^{3} \mathrm{~J}=\right.$ $\left.6.3 \mathrm{~Hz},{ }^{3} \mathrm{~J}=7.8 \mathrm{~Hz}, 1 \mathrm{H}, \mathrm{CHCH}_{3}\right), 7.7-7.95$ (m, 4H, Phthaloyl).

$$
\mathrm{C}_{14} \mathrm{HH}_{12} \mathrm{~F}_{3} \mathrm{NO}_{7} \mathrm{~S}(395.3) \quad \text { Ber. C } 42.54 \mathrm{H} 3.06 \mathrm{~N} 3.54 \mathrm{~S} 8.11
$$$$
\text { Gef. C } 42.49 \text { H } 2.88 \text { N } 3.68 \text { S } 8.16
$$

$4 \mathrm{~b}(2 R, 3 S)$ und $4 \mathrm{c}(2 S, 3 R)$ : Aus jeweils $1.69 \mathrm{~g}(6.0 \mathrm{mmol})$ TFMSA in $35 \mathrm{ml}$ Dichlormethan und $1.05 \mathrm{~g}(4.0 \mathrm{mmol}) 3 \mathrm{~b}$ bzw. $3 \mathrm{c}$ in jeweils $0.47 \mathrm{~g}(6.0 \mathrm{mmol})$ Pyridin und $15 \mathrm{ml}$ Dichlormethan; nach Aufarbeiten Ausb. $1.58 \mathrm{~g}(100 \%) 4 \mathrm{~b},[\alpha]_{D}^{20}=+46.7\left(c=1, \mathrm{CCl}_{4}\right)$ bzw. $1.58 \mathrm{~g}(100 \%) 4 \mathrm{c},[\alpha]]_{\mathrm{B}}^{20}=-44.1\left(\mathrm{c}=1, \mathrm{CCl}_{4}\right)$.

$N$-Phthaloylallothreonin-methylester 3d-f: Jeweils $1.98 \mathrm{mg}(5.0$ $\mathrm{mmol}$ ) $4 \mathrm{a}$-c werden mit $50 \mathrm{ml}$ Wasser $12 \mathrm{~h}$ bei Raumtemp. gerührt und anschließend zweimal mit jeweils $100 \mathrm{ml}$ Chloroform extrahiert. Die vereinigten organischen Phasen werden mit Magnesiumsulfat getrocknet, über Kieselgel mit PE/EE (2:1) chromatographiert und mittels MPLC gereinigt.

\section{3d (Racemat): Aus 4a; Ausb. $0.70 \mathrm{~g}(54 \%$ ) farbloses Öl.}

3e $(2 R, 3 R)$ : Aus 4 b; Ausb. $0.622 \mathrm{~g}(47 \%)$ farbloses $O \mathrm{l},[\alpha]_{\mathrm{D}}^{20}=$ $+9.5\left(\mathrm{c}=-1, \mathrm{CHCl}_{3}\right) .-{ }^{1} \mathrm{H}-\mathrm{NMR}\left(\mathrm{CDCl}_{3}\right): \delta=1.28\left(\mathrm{~d},{ }^{3} \mathrm{~J}=\right.$ $\left.6.5 \mathrm{~Hz}, 3 \mathrm{H}, \mathrm{CHCH}_{3}\right), 3.76\left(\mathrm{~s}, 3 \mathrm{H}, \mathrm{COCH}_{3}\right), 4.15(\overline{\mathrm{s}}, 1 \mathrm{H}, \mathrm{OH}), 4.56$ $\left(\mathrm{dq},{ }^{3} \mathrm{~J}=6.5 \mathrm{~Hz},{ }^{3} \mathrm{~J}=6.5 \mathrm{~Hz}, 1 \mathrm{H}, \mathrm{OCH}\right), 4.72\left(\mathrm{~d},{ }^{3} \mathrm{~J}=6.5 \mathrm{~Hz}\right.$, $1 \mathrm{H}, \mathrm{CHN}$ ), $7.74-7.92$ (m, 4H, Phthaloyl).

$$
\begin{array}{cccccc}
\mathrm{C}_{13} \mathrm{H}_{13} \mathrm{NO}_{5}(263.2) & \text { Ber. } & \text { C } 59.31 & \mathrm{H} & 4.98 & \text { N } 5.32 \\
& \text { Gef. } & \text { C } 59.16 & \text { H } 5.11 & \text { N } 5.07
\end{array}
$$

3f $(2 S, 3 S)$ : Aus 4c; Ausb. $0.98 \mathrm{~g}(75 \%)$ farbloses Öl, $[\alpha]_{\mathrm{D}}^{20}=-9.6$ (c $=1, \mathrm{CHCl}_{3}$ ).

$N$-Phthaloyl-O-(trifluormethylsulfonyl) allothreonin-methylester 4e, f: $4 \mathbf{e}(2 R, 3 R)$ : Aus $0.55 \mathrm{~g}(2.07 \mathrm{mmol}) 3 \mathrm{e}$ in $20 \mathrm{ml}$ Dichlormethan und $0.88 \mathrm{~g}$ (3.12 mmol) TFMSA in $0.25 \mathrm{~g}$ (3.16 mmol) Pyridin; nach Aufarbeiten Ausb. $0.81 \mathrm{~g}(99 \%)$ farblose Kristalle, Schmp. $109-111^{\circ} \mathrm{C}$ (Zers.), $[\alpha]_{\mathrm{D}}^{20}=+93.4\left(c=1, \mathrm{CCl}_{4}\right) .-{ }^{1} \mathrm{H}-\mathrm{NMR}$ $\left(\mathrm{CDCl}_{3}\right): \delta=1.55\left(\mathrm{~d},{ }^{3} \mathrm{~J}=7.4 \mathrm{~Hz}, 3 \mathrm{H}, \mathrm{CHCH}_{3}\right), 3.83(\mathrm{~s}, 3 \mathrm{H}$, $\left.\mathrm{OCH}_{3}\right), 5.20\left(\mathrm{~d},{ }^{3} \mathrm{~J}=5 \mathrm{~Hz}, 1 \mathrm{H}, \mathrm{NCH}\right), 5.76\left(\mathrm{dq},{ }^{3} \mathrm{~J}=5 \mathrm{~Hz},{ }^{3} \mathrm{~J}=\right.$ $7.4 \mathrm{~Hz}, 1 \mathrm{H}, \mathrm{OCH}), 7.93$ (m, 4H, Phthaloyl).

$$
\begin{array}{ll}
\mathrm{C}_{14} \mathrm{H}_{12} \mathrm{~F}_{3} \mathrm{NO}_{7} \mathrm{~S} \text { (395.3) } & \text { Ber. C 42.54 H 3.06 N } 3.54 \mathrm{~S} 8.11 \\
& \text { Gef. C 42.66 H 3.06 N } 3.53 \mathrm{~S} 7.98
\end{array}
$$

4f $(2 S, 3 S)$ : Aus $0.95 \mathrm{~g}(3.6 \mathrm{mmol}) 3 \mathrm{f}$ in $8 \mathrm{ml}$ Dichlormethan und $1.55 \mathrm{~g}$ (5.5 mmol) TFMSA in $0.435 \mathrm{~g}(5.5 \mathrm{mmol})$ Pyridin; nach Aufarbeiten Ausb. $1.41 \mathrm{~g}(99 \%)$ farblose Kristalle, Schmp. $109-111^{\circ} \mathrm{C}$ (Zers.), $[\alpha] \mathrm{B}^{0}=-93.1\left(c=1, \mathrm{CCl}_{4}\right)$.

Alkylierung von Aromaten: In einem i. Vak. mit fächelnder Flamme ausgeheizten und mit trockenem Stickstoff belüfteten Rundkolben mit aufgesetztem RückfluBkühler werden das in überschüssigem Aromat gelöste Triflat $\mathbf{4}$ und Trifluormethansulfonsäure (TFMS) $10 \mathrm{~h}$ bei $80^{\circ} \mathrm{C}$ gerührt. Anschließend wird i. Vak. der überschüssige Aromat abdestilliert, der Rückstand mit $50 \mathrm{ml}$ Wasser versetzt und mit verdünnter $\mathrm{NaHCO}_{3}$-Lösung neutralisiert. Nach dreimaligem Extrahieren mit jeweils $50 \mathrm{ml}$ Chloroform werden die vereinigten organischen Phasen mit Magnesiumsulfat getrocknet, dann wird Chloroform abdestilliert, der Rückstand über Kieselgel mit PE/EE (3:1) chromatographiert und mittels MPLC mit PE/ EE gereinigt.

1) Alkylierung von Benzol zu $\beta$-Methyl-N-phthaloylphenylalaninmethylestern 6

6d (Racemat): Aus $1.58 \mathrm{~g}$ (4.0 mmol) 4a, $0.6 \mathrm{~g}$ (4.0 mmol) TFMS

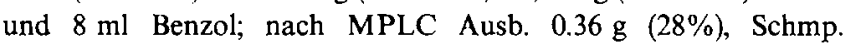
$142-143^{\circ} \mathrm{C}$. 
6e $(2 R, 3 R)$ und $6 \mathrm{~b}(2 R, 3 S)$ : a) Aus $1.58 \mathrm{~g}(4.0 \mathrm{mmol}) 4 \mathrm{~b}, 0.61 \mathrm{~g}$ (4.0 mmol) TFMS und $8 \mathrm{ml}$ Benzol; nach MPLC Ausb. $0.32 \mathrm{~g}$ $(25 \%) 6$ e, Schmp. $106.5-107 \mathrm{C},[\alpha]_{D}^{20}=+139\left(c=1, \mathrm{CHCl}_{3}\right)$. $\left.{ }^{1} \mathrm{H}-\mathrm{NMR}\left(\mathrm{CDCl}_{3}\right): \delta=1.56 \mathrm{dd},{ }^{3} \mathrm{~J}=6.85 \mathrm{~Hz}, 3 \mathrm{H}, \mathrm{CHCH}_{3}\right), 3.75$ (s, $\left.3 \mathrm{H}, \mathrm{OCH}_{3}\right), 3.98\left(\mathrm{dq},{ }^{3} \mathrm{~J}=10.85 \mathrm{~Hz},{ }^{3} \mathrm{~J}=6.5 \mathrm{~Hz}, 1 \mathrm{H}, \mathrm{CHCH}\right.$ ), $4.95\left(\mathrm{~d},{ }^{3} \mathrm{~J}=10.8 \mathrm{~Hz}, 1 \mathrm{H}, \mathrm{NCH}\right), 6.98-7.16(\mathrm{~m}, 5 \mathrm{H}$, Phenyl), $7.58-7.88(\mathrm{~m}, 4 \mathrm{H}$, Phthaloyl! $-\mathrm{MS}(70 \mathrm{eV}): m / z(\%)=323(5)$ $\left[\mathrm{M}^{+}\right], 264(6)\left[\mathrm{M}^{+}-\mathrm{CO}_{2} \mathrm{CH}_{3}\right], 176(100)\left[\mathrm{M}^{+}-\right.$Phthalimid], 105 (93) $\left[\mathrm{C}_{8} \mathrm{H}_{9}^{+}\right.$aus Benzylspaltung].

$$
\begin{array}{ll}
\mathrm{C}_{19} \mathrm{H}_{17} \mathrm{NO}_{4} \text { (323.3) } & \text { Ber. C } 70.58 \text { H } 5.30 \text { N } 4.33 \\
& \text { Gef. C } 70.55 \text { H } 5.41 \text { N } 4.08
\end{array}
$$

Ferner erhält man $0.007 \mathrm{~g}(0.5 \%)$ bb als $\mathrm{Ol},[\alpha]_{\mathrm{D}}^{20}=+121.0(\mathrm{c}=$ $\left.0.4, \mathrm{CHCl}_{3}\right) .-{ }^{1} \mathrm{H}-\mathrm{NMR}\left(\mathrm{CDCl}_{3}\right): \delta=1.20\left(\mathrm{~d},{ }^{3} \mathrm{~J}=7.15 \mathrm{~Hz}, 3 \mathrm{H}\right.$, $\left.\mathrm{CHCH}_{3}\right), 3.55\left(\mathrm{~s}, 3 \mathrm{H}, \mathrm{OCH}_{3}\right), 3.95\left(\mathrm{dq},{ }^{3} \mathrm{~J}=9.9 \mathrm{~Hz},{ }^{3} \mathrm{~J}=7.1 \mathrm{~Hz}\right.$, $\left.1 \mathrm{H}, \mathrm{CHCH}_{3}\right), 5.09\left(\mathrm{~d},{ }^{3} \mathrm{~J}=9.9 \mathrm{~Hz}, 1 \mathrm{H}, \mathrm{NCH}\right), 7.20-7.39(\mathrm{~m}, 5 \mathrm{H}$, Phenyl), 7.74-7.92 (m, $4 \mathrm{H}$, Phthaloyl). $-\mathrm{MS}(70 \mathrm{eV}): m^{\prime} z(\%)=$ 323 (3) $\left[\mathrm{M}^{+}\right], 264$ (6) $\left[\mathrm{M}^{+}-\mathrm{CO}_{2} \mathrm{CH}_{3}\right] .218$ (10) [ ${ }^{+} \mathrm{CH}-$ (NPht) $\mathrm{CO}_{2} \mathrm{CH}_{3}$ ], 176 (92) [M+ $\mathrm{M}^{+}$Phthalimid], 148 (7) [Phthalimid '], 105 (100) $\left[\mathrm{C}_{8} \mathrm{H}_{9}^{+}\right.$aus Benzylspaltung]; $\mathrm{C}_{19} \mathrm{H}_{17} \mathrm{NO}_{4}$ Ber. 323.1158 Gef. 323.1158. - Diastereomerenüberschuß (d. e.): $97.9 \%$.

b) Aus $0.765 \mathrm{~g}$ (1.95 mmol) $4 \mathrm{e}, 0.291 \mathrm{~g}(1.94 \mathrm{mmol})$ TFMS und $5 \mathrm{ml}$ Benzol; nach MPLC Ausb. $0.042 \mathrm{~g}(6.7 \%) 6 \mathrm{e}$, Schmp. $106.5^{\circ} \mathrm{C}$, $[\alpha]_{0}^{20}=+139.0\left(c=1.5, \mathrm{CHCl}_{3}\right)$ sowie $0.028 \mathrm{~g}(4.4 \%) 6 \mathrm{~b}$ als $\mathrm{Ol}$, $[\alpha]_{D}^{20}=+120.0\left(c=0.5, \mathrm{CHCl}_{3}\right)$

6f $(2 S, 3 S)$ und $6 \mathrm{c}(2 S, 3 R)$ : a) Aus $1.58 \mathrm{~g}(4.0 \mathrm{mmol}) 4 \mathrm{c}, 0.61 \mathrm{~g}(4.0$ mmol) TFMS und $8 \mathrm{ml}$ Benzol; nach MPLC Ausb. $0.297 \mathrm{~g}(23 \%)$ 6f, Schmp. $107^{\circ} \mathrm{C}(\mathrm{PE}),[\alpha]_{\mathrm{D}}^{20}=-140.0\left(\mathrm{c}=1, \mathrm{CHCl}_{3}\right)$, sowic $0.010 \mathrm{~g}(0.8 \%) 6 \mathrm{c}$ als $\mathrm{Ol},[\alpha]_{0}^{20}=-120.0\left(c=1, \mathrm{CHCl}_{3}\right)$. - d. e. $=$ $96.7 \%$

c) Aus $1.40 \mathrm{~g}(3.6 \mathrm{mmol}) 4 \mathrm{f}, 0.55 \mathrm{~g}(3.6 \mathrm{mmol})$ TFMS und $8 \mathrm{ml}$ Benzol; nach MPLC Ausb. $0.102 \mathrm{~g} \mathrm{(8.8 \% )} \mathrm{6f,} \mathrm{Schmp.} \mathrm{106"} \mathrm{C,}$ $[\alpha]_{D}^{20}=-139.0\left(\mathrm{c}=1, \mathrm{CHCl}_{3}\right)$ sowic $0.070 \mathrm{~g}(6 \%) 6 \mathrm{c}$ als $\mathrm{Ol},[\alpha]_{D}^{20}=$ $-120.5\left(c=0.6, \mathrm{CHCl}_{3}\right)$.

2) Alkylierung von 'Mesityien zu $\beta$-Methyl-N-phthaloylmesitylalanin-methylestern $\mathbf{6}^{\prime}$

6'd (Racemat): Aus $0.790 \mathrm{~g}$ (2.0 mmol) 4a, $0.30 \mathrm{~g}(2.0 \mathrm{mmol})$ TFMS und $5 \mathrm{ml}$ Mesitylen; nach MPLC Ausb. $0.442 \mathrm{~g} \mathrm{(61 \% ),}$ Schmp. $156-157^{\circ} \mathrm{C}$. $-{ }^{1} \mathrm{H}-\mathrm{NMR}\left(\mathrm{CDCl}_{3}\right): \delta=1.60\left(\mathrm{~d},{ }^{3} \mathrm{~J}=\right.$ $\left.7.1 \mathrm{~Hz}, 3 \mathrm{H}, \mathrm{CHCH}_{3}\right), 2.06,2.33$ und $2.44\left(3 \mathrm{~s}, 9 \mathrm{H}, 3 \mathrm{Mesityl}-\mathrm{CH}_{3}\right)$, $3.73\left(\mathrm{~s}, 3 \mathrm{H}, \mathrm{OCH}_{3}\right), 4.50\left(\mathrm{dq},{ }^{3} \mathrm{~J}=7.1 \mathrm{~Hz},{ }^{3} \mathrm{~J}=11.4 \mathrm{~Hz}, 1 \mathrm{H}\right.$, $\left.\mathrm{CHCH}_{3}\right), 5.38\left(\mathrm{~d},{ }^{3} \mathrm{~J}=11.4 \mathrm{~Hz}, 1 \mathrm{H}, \mathrm{NCH}\right) .6 .64(\mathrm{~s}, 2 \mathrm{H}$, Mesitylen), $7.60--7.75$ (m, $4 \mathrm{H}$, Phthaloyl!.

$$
\begin{array}{llllll}
\mathrm{C}_{22} \mathrm{H}_{23} \mathrm{NO}_{4} \text { (365.4) } & \text { Ber. C } 72.31 & \text { H } 6.34 & \mathrm{~N} & 3.83 \\
& & \text { Gef. C } 72.23 & \text { H } 6.21 & \text { N } 3.55
\end{array}
$$

6'f (2S,3S): Aus $1.96 \mathrm{~g}(5.0 \mathrm{mmol}) 4 \mathrm{c}, 0.75 \mathrm{~g}$ (5.0 mmol) TFMS und $10 \mathrm{ml}$ Mesitylen; nach MPLC Ausb. $1.15 \mathrm{~g} \mathrm{(63 \% ),} \mathrm{Schmp.}$ $129-130^{\circ} \mathrm{C},[\alpha]_{\mathrm{D}}^{20}=-75.4\left(c=1, \mathrm{CHCl}_{3}\right)$.

Bestimmung der Koaleszenztemperatur: Es wurden ${ }^{1} \mathrm{H}-\mathrm{NMR}-$ Spektren von 6' $\mathrm{f}$ in $\left[\mathrm{D}_{6}\right] \mathrm{DMSO}$ bei $90 \mathrm{C}, 100 \mathrm{C}, 110 \mathrm{C}$ und $115 \mathrm{C}$ aufgenommen. Die beiden Singuletts der Mesityl- $\mathrm{CH}_{3}$-Protonen bei $\delta=2.33$ und 2.44 (in $\mathrm{CDCl}_{3}$ ) beginnen bei $115^{\circ} \mathrm{C}$ in ein breites Singulett überzugehen. Die freie Aktivierungsenthalpie wurde nach Lit. ${ }^{32)}$ berechnet.

3) Alkylierung von Hydrochinon-dimethylether zu $\beta$-Methyl-Nphthaloyl- $\beta$-(2,5-dimethoxyphenyl)alanin-methylestern 6"

6"d (Racemat): a) In 1.2-Dichlorethan: Aus $1.98 \mathrm{~g}$ (5.0 mmol) 4a, $0.75 \mathrm{~g}(5.0 \mathrm{mmol})$ TFMS und $0.69 \mathrm{~g}(5.0 \mathrm{mmol}) \mathrm{Hydrochinon-di-}$ methylether in $20 \mathrm{ml} \mathrm{1,2-Dichlorethan;} \mathrm{nach} 2 \mathrm{~h}$ bei $60 \mathrm{C}$ und Aufarbeiten mittels MPLC Ausb. $0.2 \mathrm{~g}(10 \%)$, Schmp. 132-133 C. ${ }^{1} \mathrm{H}-\mathrm{NMR}\left(\mathrm{CDCl}_{3}\right): \delta=1.57\left(\mathrm{~d},{ }^{3} \mathrm{~J}=6.9 \mathrm{~Hz}, 3 \mathrm{H}, \mathrm{CHCH}_{3}\right), 3.652$ und $3.657\left(2 \mathrm{~s}, 6 \mathrm{H}, 2 \mathrm{Hydrochinon}-\mathrm{OCH}_{3}\right), 3.74\left(\mathrm{~s}, 3 \mathrm{H}, \mathrm{CO}_{2} \mathrm{CH}_{3}\right)$, $4.19\left(\mathrm{qd},{ }^{3} \mathrm{~J}=6.85 \mathrm{~Hz},{ }^{3} \mathrm{~J}=10.8 \mathrm{~Hz}, 1 \mathrm{H}, \mathrm{CHCH}\right), 5.25\left(\mathrm{~d},{ }^{3} \mathrm{~J}=\right.$ $10.8 \mathrm{~Hz}, 1 \mathrm{H}, \mathrm{NCH}), 6.56\left(\mathrm{dd},{ }^{4} \mathrm{~J}=2.4 \mathrm{~Hz},{ }^{3} \mathrm{~J}=9 \mathrm{~Hz}, 2 \mathrm{H}, \mathrm{Hy}-\right.$ drochinon-3,4-H), $6.70\left(\mathrm{~d},{ }^{4} J=2.2 \mathrm{~Hz}, 1 \mathrm{H}\right.$, Hydrochinon-6-H), $7.57-7.73$ (m, 4H, Phthaloyl).

$$
\begin{array}{llllll}
\mathrm{C}_{21} \mathrm{H}_{21} \mathrm{NO}_{6} \text { (383.4) } & \text { Ber. C } 65.79 \text { H } 5.52 & \text { N } 3.65 \\
& \text { Gef. C } 65.90 \text { H } 5.56 & \text { N } 3.58
\end{array}
$$

b) Ohne Lösungsmittel: Aus $1.98 \mathrm{~g}(5.0 \mathrm{mmol}) \mathbf{4 a}, 0.75 \mathrm{~g}(5.0$ mmol) TFMS und $3.50 \mathrm{~g}$ (25.0 mmol) Hydrochinon-dimethylether; nach $1.5 \mathrm{~h}$ bei $80^{\circ} \mathrm{C}$ und Aufarbeiten mittels MPLC Ausb. $0.71 \mathrm{~g}$ (37\%), Schmp. $130-132$ C.

6"f ( $2 S, 3 S)$ : Aus $1.52 \mathrm{~g}(4.0 \mathrm{mmol}) 4 \mathrm{c}, 0.60 \mathrm{~g}(4.0 \mathrm{mmol})$ TFMS

\begin{tabular}{|c|c|c|c|c|}
\hline \multirow[b]{2}{*}{$\begin{array}{c}\text { Ver- } \\
\text { bindung }\end{array}$} & \multicolumn{2}{|c|}{$\begin{array}{l}\text { Ansätze in } \\
0.5 \mathrm{ml} \mathrm{CDCl}\end{array}$} & \multicolumn{2}{|c|}{$\begin{array}{c}\left.{ }^{\prime} \mathrm{H}-\mathrm{NMR}(\mathrm{CDCl})(300) \mathrm{MHz}\right) \\
\delta(\mathrm{ppm})\end{array}$} \\
\hline & $\underset{(\mathrm{mmol})}{[\mathrm{mg}]}$ & $\begin{array}{c}\text { Eu(hfc) } \\
{[\mathrm{mg}](\mathrm{mmol})}\end{array}$ & Ohne Eu(hic), & Mit Eu(hfc), \\
\hline $\begin{array}{l}3 \mathrm{~d} \\
(2 R .3 R / \\
2 S .3 S)\end{array}$ & $\left(9.9 \times 10^{-5}\right)$ & $\begin{array}{l}10.0 \\
\left(8.4 \times 10^{-6}\right)\end{array}$ & $3.767\left(\mathrm{~s}, 3 \mathrm{H}, \mathrm{OCH}_{3}\right)$ & $\left.\begin{array}{l}3.966 \\
3.894\end{array}\right\}\left(\mathrm{d} . \mathrm{\Sigma} 3 \mathrm{H} . \mathrm{OCH}_{3}\right)$ \\
\hline $\begin{array}{l}3 f \\
(2 S, 3 S)\end{array}$ & $\begin{array}{l}25.1 \\
\left(9.6 \times 10^{-5}\right)\end{array}$ & & $3.767\left(\mathrm{~s}, 3 \mathrm{H}, \mathrm{OCH}_{3}\right)$ & $3.965\left(\mathrm{~s}, 3 \mathrm{H}, \mathrm{OCH}_{3}\right)$ \\
\hline $\begin{array}{l}6 \mathrm{~d} \\
(2 R .3 R \\
2 S .3 S)\end{array}$ & $\begin{array}{l}24.8 \\
\left(7.7 \times 10^{-5}\right)\end{array}$ & $\begin{array}{l}9.0 \\
\left(7.5 \times 10^{-8}\right)\end{array}$ & $4.9536(\mathrm{~d}, 1 \mathrm{H}, \mathrm{CHN})$ & $\left.\begin{array}{l}5.7919 \\
5.9081\end{array}\right\}(2 \mathrm{~d} . \Sigma 1 \mathrm{H} . \mathrm{CHN})$ \\
\hline $\begin{array}{l}6 f \\
(2 S, 3 S)\end{array}$ & $\left(7.7 \times 10^{-5}\right)$ & & $4.9581(\mathrm{~d}, 1 \mathrm{H}, \mathrm{CHN})$ & $5.4915(\mathrm{~d}, 1 \mathrm{H}, \mathrm{CHN})$ \\
\hline $\begin{array}{l}6^{\prime} \mathrm{d} \\
(2 R .3 R / \\
2 S .3 S)\end{array}$ & $\begin{array}{l}24.8 \\
(6.8 \times 105)\end{array}$ & $\begin{array}{l}8.0 \\
\left(6.7 \times 10^{-6}\right)\end{array}$ & $3.734\left(\mathrm{~s}, 3 \mathrm{H}, \mathrm{OCH}_{3}\right)$ & $\left.\begin{array}{l}3.748 \\
3.721\end{array}\right\}\left(\mathrm{d}, \Sigma 3 \mathrm{H}, \mathrm{OCH}_{3}\right)$ \\
\hline 6'f & $\left(6.7 \times 10^{-4}\right)$ & & $3.734\left(\mathrm{~s}, 3 \mathrm{H}, \mathrm{OCH}_{3}\right)$ & $3.773\left(\mathrm{~s}, 3 \mathrm{H}, \mathrm{OCH}_{3}\right)$ \\
\hline $\begin{array}{l}6^{\prime \prime} \mathrm{d} \\
(2 R, 3 R \prime \\
2 S .3 S)\end{array}$ & $\begin{array}{l}24.7 \\
\left(6.4 \times 10^{-5}\right)\end{array}$ & $\left(1.0 \times 10^{-5}\right)$ & $1.571\left(\mathrm{~d}, 2 \mathrm{H}, \mathrm{CHCH}_{3}\right)$ & $\left.\begin{array}{l}1.828 \\
1.790\end{array}\right\}\left(2 \mathrm{~d}, \Sigma 3 \mathrm{H} . \mathrm{CHCH}_{3}\right)$ \\
\hline $\begin{array}{l}6^{\prime \prime} f \\
(2 S, 3 S)\end{array}$ & $\begin{array}{l}24.4 \\
\left(6.3 \times 10^{-5}\right)\end{array}$ & & 1.571 (d. $3 \mathrm{H} . \mathrm{CHCH}_{3}$ ) & $1.913(\mathrm{~d}, 3 \mathrm{H} . \mathrm{CHCH})$ \\
\hline
\end{tabular}
und $3.50 \mathrm{~g}(25.0 \mathrm{mmol})$ Hydrochinon-dimethylether; nach $2 \mathrm{~h}$ bei $80{ }^{\circ} \mathrm{C}$ und Aufarbeiten mittels MPLC Ausb. $0.40 \mathrm{~g} \mathrm{(26 \% )}$ als $\mathrm{Ol}$, $[\alpha]_{D}^{20}=-106.0\left(c=1, \mathrm{CHCl}_{3}\right)$.

Tab. 6. 'H-NMR-spektroskopische Bestimmung der Enantiomerenreinheit

Rückgewinnung von TFMS: $1.58 \mathrm{~g}(4.0 \mathrm{mmol}) 4 \mathrm{e}$ und $0.6 \mathrm{~g}(4.0$ mmol) TFMS in $10 \mathrm{ml}$ Benzol werden $10 \mathrm{~h}$ bei $80^{\circ} \mathrm{C}$ gerührt. AnschlieBend gibt man $1.0 \mathrm{~g}$ Bariumcarbonat und $5 \mathrm{ml}$ Wasser $\mathrm{zu}$. rührt $2 \mathrm{~h}$ bei Raumtemp. und schüttelt dann mit $150 \mathrm{ml}$ Wasser und $100 \mathrm{ml}$ Chloroform kräftig aus. Die Chloroformphase wird mit $100 \mathrm{ml} \mathrm{Wasser,} \mathrm{die} \mathrm{wäßrige} \mathrm{Phase} \mathrm{mit} 100 \mathrm{ml}$ Chloroform extrahiert. Die vereinigten organischen Phasen werden mit Magnesiumsulfat getrocknet, überschüssiges Benzol wird gemeinsam mit Chloroform i. Vak. abdestilliert und der Rückstand wie bei der Alkylierung von Aromaten beschrieben aufgearbeitet. Die vereinigten wäßrigen Phasen werden filtriert und i. Vak. eingeengt. Der farblose, kristalline Rückstand wird i. Vak. (Olpumpe) scharf getrocknet und mit $150 \mathrm{ml}$ Aceton versetzt. Nach Abfiltrieren vom Unlöslichen und Einengen des Filtrats im Rotationsverdampfer bleiben $1.72 \mathrm{~g}$ $\left(98.5 \%\right.$ ) Barium-trifluormethansulfonat (ca. $3.95 \mathrm{mmol} \mathrm{CF}_{3} \mathrm{SO}_{3}$ ) zurück.

$N$-Acetyl- $\beta$-methyl-(2S,3S)-phenylalanin (8): Die Lösung von $0.80 \mathrm{~g}(2.47 \mathrm{mmol}) 6 \mathrm{f}$ in $24 \mathrm{ml}$ Aceton wird mit $16 \mathrm{ml}$ Wasser und $5 \mathrm{ml}$ konz- $\mathrm{HCl}$ versetzt, $60 \mathrm{~h}$ bei $80^{\circ} \mathrm{C}$ gerührt und im Rotationsverdampfer eingeengt. Der Rückstand wird nach Zugeben einer gesättigten $\mathrm{Na}_{2} \mathrm{CO}_{3}$-Lösung in Wasser zweimal mit jeweils $100 \mathrm{ml}$ EE ausgeschüttelt, die wäßrige Phase mit $6 \mathrm{~N} \mathrm{HCl}$ bis zur stark 
sauren Reaktion versetzt und dreimal mit $100 \mathrm{ml} \mathrm{EE}$ ausgeschüttelt. Die vereinigten EE-Phasen werden mit Magnesiumsulfat getrocknet und eingeengt. Der kristalline Rückstand $(0.56 \mathrm{~g})$ wird nach Lit. $^{25)}$ in $22.1 \mathrm{ml}$ 2-Propanol und $3.75 \mathrm{ml} \mathrm{Wasser} \mathrm{gelöst,} \mathrm{die} \mathrm{Lösung}$ vorsichtig mit $0.46 \mathrm{~g}(12.4 \mathrm{mmol}) \mathrm{NaBH}_{4}$ versetzt, 2 h bei Raumtemp. gerührt, mit $2.6 \mathrm{ml}$ Essigsäure versetzt und $3 \mathrm{~h}$ bei $80^{\circ} \mathrm{C}$ sowie $12 \mathrm{~h}$ bei Raumtemp. gerührt. Anschließend wird das Reaktionsgemisch einrotiert, der farblose, kristalline Rückstand in $6 \mathrm{~N} \mathrm{HCl}$ aufgenommen, die Lösung mit EE extrahiert und die wäßrige Phase i. Vak. zur Trockne einrotiert. Der farblose Rückstand wird in $15 \mathrm{ml} 1 \mathrm{~N} \mathrm{NaOH}$ gelöst, die Lösung unter Eiskühlung mit $0.86 \mathrm{ml}$ Essigsäureanhydrid und $7.5 \mathrm{ml}$ Natronlauge versetzt, $1 \mathrm{~h}$ gerührt und mit konz. $\mathrm{HCl}$ auf $\mathrm{pH}<1$ angesäuert. Nach zweimaligem Extrahieren mit jeweils $100 \mathrm{ml} \mathrm{EE}$ werden die vereinigten organischen Phasen mit Magnesiumsulfat getrocknet und einrotiert; Ausb. $0.30 \mathrm{~g}(55 \%)$, Schmp. $190-192^{\circ} \mathrm{C}$ (Ethnaol/Wasser) (Lit. ${ }^{16)}$ $\left.188-190^{\circ} \mathrm{C}\right),[\alpha]_{\mathrm{D}}^{20}=+37.4(c=1, \mathrm{EtOH}, 96$ proz. $)\left(\right.$ L it. $^{16)}[\alpha]_{\mathrm{D}}^{20}=$ +36.5 (96proz. EtOH). ${ }^{1} \mathrm{H}-\mathrm{NMR}$-spektroskopisch identisch mit Lit. ${ }^{16)}$

$$
\begin{array}{llllll}
\mathrm{C}_{12} \mathrm{H}_{15} \mathrm{NO}_{3} \text { (221.2) } & \text { Ber. } \mathrm{C} 65.14 & \text { H } 6.83 & \text { N } 6.23 \\
& \text { Gef. C } 65.37 & \text { H } 6.89 & \text { N } 6.24
\end{array}
$$

4) ,Eintopfmethode“ zur Darstellung von $6 \mathbf{f}$. - a) ${ }^{t} H-N M R$-spektroskopische Bestimmung der Produktverteilung: $1.58 \mathrm{~g}(6.0 \mathrm{mmol})$ 3c und $1.69 \mathrm{~g}(6.0 \mathrm{mmol})$ TFMSA (30 ml einer $0.2 \mathrm{M}$ Lösung in Benzol) werden unter Feuchtigkeitsausschluß bei $80^{\circ} \mathrm{C}$ gerührt. In Abständen von $2 \mathrm{~h}$ wird jeweils $1 \mathrm{ml}$ entnommen, mit $10 \mathrm{ml} \mathrm{Me}-$ thanol/Wasser versetzt und mit verdünnter $\mathrm{NaHCO}_{3}$-Lösung neutralisiert. Nach Ausschütteln mit Dichlormethan wird die organische Phase mit Magnesiumsulfat getrocknet und nach Abtrennen des Lösungsmittels die prozentuale Produktverteilung an 3c, 6f, 9 und $10{ }^{1} \mathrm{H}$-NMR-spektroskopisch ermittelt (s. Tab. 4). Die ${ }^{1} \mathrm{H}-$ NMR-Spektren von $3 \mathrm{c}, 6 \mathrm{f}$ und $\mathbf{9}^{33)}$ waren bekannt, der bislang nicht bekannte $N$-Phthaloyl-vinylglycin-methylester (10) wurde zum Vergleich dargestellt aus $1.58 \mathrm{~g}(4.0 \mathrm{mmol}) 4 \mathrm{c}$ und $0.6 \mathrm{~g}(4.0 \mathrm{mmol})$ TFMS in $8 \mathrm{ml} \mathrm{Benzol;} \mathrm{Ausb.} \mathrm{nach} 10 \mathrm{~h}$ bei $80^{\circ} \mathrm{C}$ und üblicher Aufarbeitung $140.0 \mathrm{mg}(11 \%) 6$ f und $45.0 \mathrm{mg}(5 \%) \mathrm{10}$. - ${ }^{1} \mathrm{H}-\mathrm{NMR}$ $\left(\mathrm{CDCl}_{3}\right)$ von 10: $\delta=3.78\left(\mathrm{~s}, 3 \mathrm{H}, \mathrm{CO}_{2} \mathrm{CH}_{3}\right), 5.30-5.43(\mathrm{~m}, 3 \mathrm{H}$, $\left.\mathrm{CH}_{2}+\mathrm{NCH}\right), 6.33-6.44\left(\mathrm{dq}, 1 \mathrm{H}, \mathrm{CH}_{2}=\mathrm{CH}\right) .7 .74-7.90(\mathrm{~m}, 4 \mathrm{H}$, Phthaloyl). - MS (70 eV): $m / z(\%)=245(7)\left[\mathrm{M}^{+}\right], 213\left[\mathrm{M}^{+}\right.$ - $\left.\mathrm{CH}_{4} \mathrm{O}\right], 186\left[\mathrm{M}^{+}-\mathrm{COCH}_{3}\right] ; \mathrm{C}_{13} \mathrm{H}_{11} \mathrm{NO}_{4}$ Ber. $245.0689 \mathrm{Gef}$. 245.0689 .

b) Präparativer Ansatz: $0.53 \mathrm{~g}(2.0 \mathrm{mmol}) 3 \mathrm{c}$ und $0.56 \mathrm{~g}(2.0$ mmol) TFMSA werden in $6 \mathrm{ml}$ Benzol bei Raumtemp. vorgelegt und unter Feuchtigkeitsausschluß $10 \mathrm{~h}$ bei $80^{\circ} \mathrm{C}$ gerührt; Ausb. nach üblicher Aufarbeitung $0.15 \mathrm{~g} \mathrm{(23 \% )} 6 \mathrm{f},[\alpha]_{\mathrm{D}}^{20}=-138.3(\mathrm{c}=$ 1.1, $\mathrm{CHCl}_{3}$ ). - c) Rückgewinnung von TFMS: Aus der Umsetzung von $0.53 \mathrm{~g}$ (4.0 mmol) $3 \mathrm{c}$ mit $1.31 \mathrm{~g}(4.0 \mathrm{mmol})$ TFMSA in $5 \mathrm{ml}$ Benzol werden nach $10 \mathrm{~h}$ bei $80^{\circ} \mathrm{C}$ und Aufarbeiten wie bei der Alkylierung von Benzol mit 4e/TFMS beschrieben $1.71 \mathrm{~g}(97 \%)$

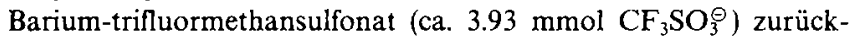
gewonnen.

5) $\mathrm{AlCl}_{3}$-katalysierte Alkylierung von Benzol: In einem Rundkolben mit aufgesetztem Tropftrichter gibt man unter Rühren zu $4 \mathrm{ml}$ Benzol $0.266 \mathrm{~g}(2.0 \mathrm{mmol})$ bzw. $0.53 \mathrm{~g}(4.0 \mathrm{mmol})$ Aluminiumchlorid und tropft anschließend die Iösung von $0.79 \mathrm{~g}$ (2.0 mmol) $4 \mathrm{c}$ in $4 \mathrm{ml}$ Benzol langsam zu. Hierbei verfärbt sich das Reaktionsgemisch von Gelb über Orange nach Rot. Nach $3 \mathrm{~h}$ schüttelt man mit Wasser aus, extrahiert die wäßrige Phase zweimal mit jeweils $30 \mathrm{ml}$ Benzol, trocknet die vereinigten organischen Phasen mit Magnesiumsulfat, chromatographiert über eine Kieselgelsäule mit PE/EE (3:1) und trennt die Produkte $6 \mathrm{c}$ und $6 \mathrm{f}$ anschließend mittels MPLC.
Tab. 7. $\mathrm{AlCl}_{3}$-katalysierte Alkylierung von Benzol mit $\mathrm{N}$-Phthaloyl$O$-(trifluormethyisulfonyl)-(2S,3R)-threonin-methylester (4c) $\mathrm{zu}$ threo- $(6 \mathrm{c})$ und erythro- $\beta$-Methyl- $N$-phthaloylphenylalanin-methyl-

\begin{tabular}{|c|c|c|c|c|c|c|c|}
\hline $\begin{array}{l}\text { Einge- } \\
\text { setzte } \\
\text { Mengen } \\
\text { 4c: } \mathrm{AlCl}_{3}\end{array}$ & $\begin{array}{l}\text { Reakt.- } \\
\text { Temp. } \\
{\left[{ }^{\circ} \mathrm{C}\right]}\end{array}$ & $\begin{array}{c}\text { Ausb. } \\
\text { [mg] } \\
(\%)\end{array}$ & $\begin{array}{c}\mathbf{6 c} \\
[x]]_{0} \\
(\mathrm{CHCl})\end{array}$ & $\begin{array}{c}+ \\
e . e^{2\}} \\
(\%)\end{array}$ & $\begin{array}{c}\text { Ausb. } \\
{[\mathrm{mg}]} \\
(\%)\end{array}$ & $\begin{array}{c}\mathbf{6 f} \\
{[\alpha]^{8}} \\
\left(\mathrm{CHCl}_{3}\right)\end{array}$ & $\begin{array}{l}\text { e.e. } e^{a)} \\
(\%)\end{array}$ \\
\hline $1: 2$ & Raumtemp. & $\begin{array}{l}100 \\
(12)\end{array}$ & $\begin{array}{l}-105 \\
(c=1.5)\end{array}$ & 87 & & & \\
\hline $1: 2$ & Raumtemp. & $\begin{array}{l}65 \\
(10)\end{array}$ & $\begin{array}{l}-115.75 \\
(c=0.4)\end{array}$ & 94 & $\begin{array}{l}85 \\
(13)\end{array}$ & $\begin{array}{l}-122 \\
(c=0.16)\end{array}$ & 84 \\
\hline $1: 2$ & 40 & $\begin{array}{l}75 \\
(12)\end{array}$ & $\begin{array}{l}-114 \\
(c=0.4)\end{array}$ & 93 & $\begin{array}{l}75 \\
(12)\end{array}$ & $\begin{array}{l}-120 \\
(c=0.26)\end{array}$ & 86 \\
\hline $1: 2$ & 80 & & & & $\begin{array}{l}40 \\
(6)\end{array}$ & $\begin{array}{l}-75 \\
(c=0.7)\end{array}$ & 53 \\
\hline
\end{tabular}
estern (6f)

a) Berechnet aus $[\alpha]_{\mathrm{D}}^{20}$ für $6 \mathrm{c}(2 S, 3 R)=-120$ und für $6 \mathrm{f}(2 S, 3 S)=$ -140 nach Lit. $^{37}$.

6) Alkylierung von Benzol mit O-Methylsulfonyl- $N$-phthaloyl(2R,3S)-bzw. (2S,3R)-threonin-methylester

$O$-Methylsulfonyl-N-phthaloylthreonin-methylester: a) $(2 R, 3 S)$ : In einem Rundkolben tropft man unter Feuchtigkeitsausschluß bei $-20^{\circ} \mathrm{C}$ unter Rühren zu $1.32 \mathrm{~g}(5.0 \mathrm{mmol}) \mathbf{3 b}$ in $20 \mathrm{ml}$ Dichlormethan langsam $0.86 \mathrm{~g}$ (7.5 mmol) Methansulfonylchlorid (MSC) und anschließend $0.76 \mathrm{~g}$ ( $7.5 \mathrm{mmol})$ Triethylamin $\mathrm{zu}$, läßt dann das Reaktionsgemisch auf Raumtemp. erwärmen und destilliert das Lösungsmittel i. Vak. ab. Man gibt zum Rückstand $200 \mathrm{ml}$ Diethylether, schüttelt die Lösung zweimal mit jeweils $100 \mathrm{ml}$ Wasser, danach mit $100 \mathrm{ml}$ verd. $\mathrm{HCl}$ und nochmals zweimal mit jeweils $100 \mathrm{ml}$ Wasser aus. Nach Trocknen der organischen Phase mit Magnesiumsulfat wird das Lösungsmittel abdestilliert und der Rückstand über eine Kieselgelsäule mit PE/EE (3:2) chromatographiert; Ausb. $0.62 \mathrm{~g}(36 \%)$, Schmp. $89^{\circ} \mathrm{C},[\alpha]_{\mathrm{D}}^{20}=+48.5(c=1.2$, $\mathrm{CCl}_{4}$ ).

b) $(2 S, 3 R)$ : Wie vorstehend beschrieben aus $1.32 \mathrm{~g}(5.0 \mathrm{mmol}) 3 \mathrm{c}$, $0.86 \mathrm{~g}(7.5 \mathrm{mmol}) \mathrm{MSC}, 0.76 \mathrm{~g}(7.5 \mathrm{mmol})$ Triethylamin und $30 \mathrm{ml}$ Dichlormethan; Ausb. $0.86 \mathrm{~g}(50 \%)$, Schmp. $88-90^{\circ} \mathrm{C}$ (Tetrachlorkohlenstoff $),[\alpha]_{\mathrm{D}}^{20}=-49.75\left(c=1, \mathrm{CCl}_{4}\right) .-{ }^{1} \mathrm{H}-\mathrm{NMR}\left(\mathrm{CDCl}_{3}\right)$ : $\delta=1.60\left(\mathrm{~d},{ }^{3} \mathrm{~J}=6.4 \mathrm{~Hz}, 3 \mathrm{H}, \mathrm{CHCH}_{3}\right), 3.02\left(\mathrm{~s}, 3 \mathrm{H}, \mathrm{SO}_{2} \mathrm{CH}_{3}\right), 3.77$ (s, $\left.3 \mathrm{H}, \mathrm{CO}_{2} \mathrm{CH}_{3}\right), 4.88\left(\mathrm{~d},{ }^{3} \mathrm{~J}=5.6 \mathrm{~Hz}, 1 \mathrm{H}, \mathrm{CHN}\right), 5.59\left(\mathrm{dq},{ }^{3} \mathrm{~J}=\right.$ $\left.5.6 \mathrm{~Hz},{ }^{3} J=6.4 \mathrm{~Hz}, 1 \mathrm{H}, \mathrm{CHCH}_{3}\right), 7.85(\mathrm{~m}, 4 \mathrm{H}$, Phthaloyl).

$$
\begin{array}{lllllll}
\mathrm{C}_{14} \mathrm{H}_{45} \mathrm{NO}_{7} \mathrm{~S}(341.3) & \text { Ber. } & \mathrm{C} 49.26 & \mathrm{H} 4.43 & \mathrm{~N} 4.10 & \mathrm{~S} 9.39 \\
& \text { Gef. } & \mathrm{C} 49.26 & \text { H } 4.42 & \text { N } 4.20 & \text { S } 9.32
\end{array}
$$

c) Die Alkylierung von Benzol erfolgt wie bei der Limsetzung von Benzol mit $4 b / T F M S$ beschrieben.

6e $(2 R, 3 R)$ : Aus $0.621 \mathrm{~g}(1.82 \mathrm{mmol}) O$-Methylsulfonyl- $N$-phthaloyl-(2R,3S)-threonin-methylester und $0.273 \mathrm{~g}(1.82 \mathrm{mmol}) \mathrm{TFMS}$ in $5 \mathrm{ml} \mathrm{Benzol;}$ nach $10 \mathrm{~h}$ bei $80^{\circ} \mathrm{C}$ und Aufarbeiten Ausb. $32.0 \mathrm{mg}$ $(5.4 \%)$, Schmp. $107^{\circ} \mathrm{C},[\alpha]_{\mathrm{D}}^{20}=+138.7\left(c=0.5, \mathrm{CHCl}_{3}\right)$.

6f $(2 S, 3 S)$ : Aus $0.56 \mathrm{~g}$ (1.64 mmol) $O$-Methylsulfonyl- $N$-phthaloyl-(2S,3R)-threonin-methylester und $0.25 \mathrm{~g}$ (1.64 mmol) TFMS in $5 \mathrm{ml} \mathrm{Benzol;}$ nach $10 \mathrm{~h}$ bei $80^{\circ} \mathrm{C}$ und Aufarbeiten Ausb. $27.0 \mathrm{mg}$ $(6 \%)$, Schmp. $106.5-107^{\circ} \mathrm{C},[\alpha]_{\mathrm{D}}^{20}=-140\left(c=0.5, \mathrm{CHCl}_{3}\right)$.

7) Versuche zur Alkylierung von Benzol mit $\beta$-Chlor- $\beta$-methyl-Nphthaloyl-(2S,3R)-alanin-methylester

$\beta$-Chlor- $\beta$-methyl- $N$-phthaloyl-(2S,3R)-alanin-methylester: Unter Feuchtigkeitsausschlu $\beta$ werden $2.63 \mathrm{~g}(10.0 \mathrm{mmol}) 3 \mathrm{c}$ und $5 \mathrm{ml}$ Thionylchlorid $15 \mathrm{~h}$ unter Rückfluß gerührt, wobei sich das Reaktionsgemisch orange-gelb verfärbt. Anschließend wird überschüssiges Thionylchlorid i. Vak. abdestilliert und der Rückstand in 
$100 \mathrm{ml}$ Chloroform gelöst. Nach zweimaligem Ausschütteln mit jeweils $100 \mathrm{ml}$ verdünnter $\mathrm{NaHCO}_{3}$-Lösung wird die organische Phase mit Magnesiumsulfat getrocknet, über Kieselgel mit PE/EE (3:1) chromatographiert und das gelbliche Produkt aus PE umkristallisiert; Ausb. 2.0 g (71\%), Schmp. $113-114^{\circ} \mathrm{C}$. $-{ }^{1} \mathrm{H}-\mathrm{NMR}$ $\left(\mathrm{CDCl}_{3}\right): \delta=1.82\left(\mathrm{~d},{ }^{3} \mathrm{~J}=6.3 \mathrm{~Hz}, 3 \mathrm{H}, \mathrm{CHCH}_{3}\right), 3.75(\mathrm{~s}, 3 \mathrm{H}$, $\left.\mathrm{CO}_{2} \mathrm{CH}_{3}\right), 4.85\left(\mathrm{~d},{ }^{3} \mathrm{~J}=9.7 \mathrm{~Hz}, 1 \mathrm{H}, \mathrm{NCH}\right), 4.92-4.99$ (qd, ${ }^{3} \mathrm{~J}=$ $\left.9.7 \mathrm{~Hz},{ }^{3} \mathrm{~J}=6.3 \mathrm{~Hz}, 1 \mathrm{H}, \mathrm{CHCH}_{3}\right), 7.76-7.91(\mathrm{~m}, 4 \mathrm{H}$, Phthaloyl).

$$
\begin{array}{lllllll}
\mathrm{C}_{13} \mathrm{H}_{12} \mathrm{ClNO}_{4}(281.7) & \text { Ber. } \mathrm{C} 55.43 & \mathrm{H} 4.29 & \mathrm{Cl} 12.58 & \mathrm{~N} 4.97 \\
& \text { Gef. C } 55.53 & \mathrm{H} 4.30 & \mathrm{Cl} 12.56 & \text { N } 4.92
\end{array}
$$

Unter Eiskühlung und Rühren werden zu einer Suspension von Aluminium-oder Eisenchlorid (4.0 oder $10.0 \mathrm{mmol}$ ) in $6 \mathrm{ml}$ Benzol $0.563 \mathrm{~g}$ ( $2.0 \mathrm{mmol}) \beta$-Chor- $\beta$-methyl- $N$-phthaloyl- $(2 S, 3 R$-alaninmethylester getropft, dann wird unter Feuchtigkeitsausschluß $18 \mathrm{~h}$ bei Raumtemp., $60^{\circ} \mathrm{C}$ oder $80^{\circ} \mathrm{C}$ gerührt. Anschließend wird mit ${ }_{1} 5 \mathrm{ml}$ Eis/Wasser ausgeschüttelt, die organische Phase abgetrennt, mit Magnesiumsulfat getrocknet und nach Abdestillieren von überschüssigem Benzol das Reaktionsgemisch 'H-NMR-spektroskopisch untersucht. Es konnte kein Alkylierungsprodukt 6 nachgewiesen werden.

8) Alkylierung von Benzol mit (R)- und (S)-3-(Trifluormethylsulfonyloxy)buttersäure-methylestern 12. $-(R)-12$ und (S)-12: Aus jeweils $0.50 \mathrm{~g}(4.6 \mathrm{mmol})(R)-(-)$ - und $(S)-(+)-3$-Hydroxybuttersäure-methylester (11) (Fa. EGA), $1.67 \mathrm{~g}$ (5.9 mmol) TFMSA und $0.47 \mathrm{~g}(5.9 \mathrm{mmol})$ Pyridin in $40 \mathrm{ml}$ Dichlormethan wie bei der Darstellung der Triflate 4 beschrieben: Ausb. $0.8 \mathrm{~g} \mathrm{(76 \% )}(R)-12$ als farbloses Ol, $[\alpha]_{D}^{20}=-2.4\left(c=1.25, \mathrm{CHCl}_{3}\right)$.- ${ }^{\prime} \mathrm{H}-\mathrm{NMR}$ $\left(\mathrm{CDCl}_{3}\right): \delta=1.62\left(\mathrm{~d},{ }^{3} \mathrm{~J}=6.4 \mathrm{~Hz}, 3 \mathrm{H}, \mathrm{CHCH}_{3}\right), 2.81\left(\mathrm{dq},{ }^{3} J_{\mathrm{H}^{2}, \mathrm{H}^{\mathrm{c}}}=\right.$ $\left.7.3 \mathrm{~Hz},{ }^{3} J_{\mathrm{H}^{\mathrm{b}} . \mathrm{H}^{\mathrm{c}}}=5.7 \mathrm{~Hz},{ }^{2} J_{\mathrm{H}^{\mathrm{a}}, \mathrm{H}^{\mathrm{b}}}=-16.4 \mathrm{~Hz}, 2 \mathrm{H}, \mathrm{CHCH}^{\mathrm{a}} \mathrm{H}^{\mathrm{b}}\right), 3.79$ (s, $\left.3 \mathrm{H}, \mathrm{OCH}_{3}\right), 5.43\left(\mathrm{~m},{ }^{3} \mathrm{~J}=6.4 \mathrm{~Hz}, 1 \mathrm{H}, \mathrm{CHCH}_{3}\right)$.

\section{$\mathrm{C}_{6} \mathrm{H}_{9} \mathrm{~F}_{3} \mathrm{O}_{5} \mathrm{~S}(250.2) \quad$ Ber. C $28.80 \mathrm{H} 3.63 \mathrm{~S} 12.81$ Gef. C 28.85 H 3.85 S 12.69}

Ferner erhält man $0.93 \mathrm{~g}(87 \%)(S)-12$ als farbloses $\delta 1,[\alpha]_{\mathrm{D}}^{20}=$ $+2.5\left(c=2, \mathrm{CHCl}_{3}\right)$.

3-Phenylbuttersäure-methylester (13) (Racemat): a) Aus $0.82 \mathrm{~g}$ $(3.30 \mathrm{mmol})(R)-12$ und $0.49 \mathrm{~g}(3.30 \mathrm{mmol}) \mathrm{TFMS}$ in $6 \mathrm{ml}$ Benzol wie bei der Alkylierung von Benzol mit 4 beschrieben; nach Trocknen mit Magnesiumsulfat, Abdestillieren des Lösungsmittels und Kugelrohrdestillation des Rückstandes bei Sdp. $120-122^{\circ} \mathrm{C} /$ 15 Torr Ausb. $0.19 \mathrm{~g}(32 \%)$, farbloses Öl, $[\alpha]_{\mathrm{D}}^{20}=+2.13(c=1$, $\left.\mathrm{C}_{6} \mathrm{H}_{6}\right)\left(\mathrm{Lit}^{34}{ }^{34}\right.$ für $(R)-13[\alpha]_{\mathrm{D}}^{20}=+44.3\left(\mathrm{C}_{6} \mathrm{H}_{6}\right)$. - b) Aus $0.90 \mathrm{~g}(3.59$ mmol) (S)-12 und $0.54 \mathrm{~g}$ (3.59 mmol) TFMS in $8 \mathrm{ml}$ Benzol wie vorstehend beschrieben; Ausb. $0.41 \mathrm{~g}(64 \%)$, farbloses $\mathrm{Ol},[\alpha]_{\mathrm{D}}^{20}=$ $-0.6\left(c=1.7, \mathrm{C}_{6} \mathrm{H}_{6}\right)$.

9) Alkylierung von Benzol mit 1-Phthalimido-2-(trifluormethylsulfonyloxy)propan (16). - 1-Phthalimido-2-propanole 15: Man gibt bei Raumtemp. unter Rühren zu der Lösung von $N$-(Ethoxycarbonyl)phthalimid in Acetonitril langsam das 1-Aminopropanol 14 zu, wobei leichte Erwärmung erfolgt, rührt $12 \mathrm{~h}$ bei Raumtemp., destilliert das Lösungsmittel i. Vak. ab, gibt zum Rückstand $150 \mathrm{ml}$ Chloroform. schüttelt die Lösung dreimal mit jeweils $50 \mathrm{ml}$ verd. $\mathrm{HCl}$ und anschließend zweimal mit jeweils $100 \mathrm{ml}$ Wasser aus, trocknet die Chloroform-Lösung mit Magnesiumsulfat, destilliert dann Chloroform i. Vak. ab und kristallisiert den farblosen Rückstand aus PE/EE (1:1) um.

(R)-15: Aus $0.96 \mathrm{~g}(12.8 \mathrm{mmol})(R)-14\left\langle[\alpha]_{\mathrm{D}}^{20}=-24.8(c=1.2\right.$, EtOH $)$, e.e. $=95 \%\left(\right.$ Lit. $\left.^{35)}-25.8(c=1.7, \mathrm{EtOH})\right\rangle$ und $2.68 \mathrm{~g}$ (13.2 mmol) $N$-(Ethoxycarbonyl)phthalimid in $20 \mathrm{ml}$ Acetonitril nach Reinigung durch Säulenchromatographie mit PE/EE (1:1);
Ausb. $1.5 \mathrm{~g}(57 \%)$, Schmp. $105-106^{\circ} \mathrm{C},[\alpha]_{\mathrm{D}}^{20}=-31.46(c=1.6$, $\mathrm{CHCl}_{3}$ ).

$$
\begin{array}{lllll}
\mathrm{C}_{11} \mathrm{H}_{11} \mathrm{NO}_{3} \text { (205.2) } & \text { Ber. } \mathrm{C} 64.38 & \text { H } 5.40 & \text { N } 6.82 \\
& \text { Gef. C } 64.19 & \text { H } 5.41 & \text { N } 6.74
\end{array}
$$

(S)-15: Aus $0.96 \mathrm{~g}(12.8 \mathrm{mmol})(S)-14\left\langle[\alpha]_{0}^{20}=+24.6(c=1.2\right.$, $\mathrm{EtOH})$, e.e. $=95.3 \%>$ und $2.64 \mathrm{~g}(13.0 \mathrm{mmol}) \mathrm{N}$-(Ethoxycarbonyl)phthalimid in $10 \mathrm{ml}$ Acetonitril; Ausb. $2.15 \mathrm{~g} \mathrm{(82 \% ),} \mathrm{Schmp.}$ $105^{\circ} \mathrm{C}$ (nach chromatographischer Reinigung), $[\alpha]_{D}^{20}=+29.9(c=$ 1.3, $\mathrm{CHCl}_{3}$ ).

1-Phthalimido-2-(trifluormethylsulfonyloxy) propane

(16): Die Darstellung erfolgt analog der der Triflate 4.

(R)-16: Aus $1.0 \mathrm{~g}(4.87 \mathrm{mmol})(R)-15,1.92 \mathrm{~g}(6.80 \mathrm{mmol})$ TFMSA und $0.54 \mathrm{~g}(6.82 \mathrm{mmol})$ Pyridin in $60 \mathrm{ml}$ Dichlormethan; Ausb. $1.60 \mathrm{~g}(97 \%)$, Schmp. $103.5^{\circ} \mathrm{C},[\alpha]_{\mathrm{D}}^{20}=-12.1\left(\mathrm{c}=1.4, \mathrm{CHCl}_{3}\right)$.

(S)-16: Aus $1.03 \mathrm{~g}(5.0 \mathrm{mmol})(S)-15,1.98 \mathrm{~g}(7.0 \mathrm{mmol})$ TFMSA und $0.55 \mathrm{~g}$ (7.0 mmol) Pyridin in $60 \mathrm{ml}$ Dichlormethan; nach chromatographischer Reinigung über Kieselgel mit PE/EE (3:1) Ausb. $1.65 \mathrm{~g}(98 \%)$, Schmp. $101-103^{\circ} \mathrm{C},[\alpha]_{\mathrm{D}}^{20}=+12.6(c=1$, $\left.\mathrm{CHCl}_{3}\right) .-{ }^{1} \mathrm{H}-\mathrm{NMR}\left(\mathrm{CDCl}_{3}\right): \delta=1.61\left(\mathrm{~d},{ }^{3} \mathrm{~J}=6.43 \mathrm{~Hz}, 3 \mathrm{H}, \mathrm{CH}_{3}\right)$, $3.83\left(\mathrm{dd},{ }^{3} J_{\mathrm{H}^{2}, \mathrm{H}^{c}}=3.3 \mathrm{~Hz},{ }^{2} J_{\mathrm{H}^{2}, \mathrm{H}^{\mathrm{b}}}=-14.7 \mathrm{~Hz}, 1 \mathrm{H}, \mathrm{CH}^{\mathrm{c}} \mathrm{CH}^{\mathrm{a}} \mathrm{H}^{\mathrm{b}}\right)$, $4.12\left(\mathrm{dd},{ }^{3} J_{\mathrm{H}^{\mathrm{b}}, \mathrm{H}^{\mathrm{c}}}=8.4 \mathrm{~Hz},{ }^{2} J_{\mathrm{H}^{\mathrm{a}}, \mathrm{H}^{\mathrm{b}}}=-14.7 \mathrm{~Hz}, 1 \mathrm{H}, \mathrm{CH}^{\mathrm{c}}, \mathrm{CH}^{\mathrm{a}} \mathrm{H}^{\mathrm{b}}\right)$, $5.33\left(\mathrm{~m}, 1 \mathrm{H}, \mathrm{CH}^{\mathrm{c}}\right), 7.90(\mathrm{~m}, 4 \mathrm{H}$, Phthaloyl).

\section{$\mathrm{C}_{12} \mathrm{H}_{10} \mathrm{~F}_{3} \mathrm{NO}_{5} \mathrm{~S}$ (337.2) Ber. C 42.74 H 2.99 N 4.15 S 9.50 Gef. C 42.02 H 2.88 N 4.07 S 9.65}

$\beta$-Methyl-N-phthaloyl-phenethylamine 17: Analog der Alkylierung von Benzol mit 4.

Enantiomerengemische 17: $(S / R)-17$ : Aus $1.60 \mathrm{~g}(4.75 \mathrm{mmol})(R)$ 16 und $0.71 \mathrm{~g}(4.74 \mathrm{mmol})$ TFMS in $10 \mathrm{ml}$ Benzol; nach $10 \mathrm{~h}$ bei $80^{\circ} \mathrm{C}$ Ausb. $0.67 \mathrm{~g}(54 \%)$, Schmp. $50^{\circ} \mathrm{C},[\alpha]_{\mathrm{D}}^{20}=-37.6(\mathrm{c}=1.3$, $\mathrm{CHCl}_{3}$ ).

(R/S)-17: a) Aus $1.60 \mathrm{~g}(4.75 \mathrm{mmol})(S)-16$ und $0.71 \mathrm{~g}(4.74 \mathrm{mmol})$ TFMS in $10 \mathrm{ml}$ Benzol; nach $10 \mathrm{~h}$ bei $80^{\circ} \mathrm{C}$ Ausb. $0.7 \mathrm{~g}(55 \%)$, Schmp. $49-51^{\circ} \mathrm{C},[\alpha]_{\mathrm{D}}^{20}=+38.7\left(c=1.75, \mathrm{CHCl}_{3}\right)$.

b) Aus $1.40 \mathrm{~g}$ (4.15 mmol) (S)-16 und $0.62 \mathrm{~g}(4.13 \mathrm{mmol})$ TFMS in $15 \mathrm{ml}$ Benzol; nach $40 \mathrm{~h}$ bei $40^{\circ} \mathrm{C}$ Ausb. $0.55 \mathrm{~g}(45 \%),[\alpha]_{\mathrm{D}}^{20}=$ $+58.5\left(c=1, \mathrm{CHCl}_{3}\right),(R):(S)=78: 22$ (berechnet aus $[\alpha]_{\mathrm{D}}^{20}=$ +38.7 für $(R / S)-17)$

B-Methylphenethylamine 18: $(S / R)-18: 0.67 \mathrm{~g}(2.53 \mathrm{mmol})(S / R)$ $17\left([\alpha]_{0}^{20}=-37.6\right)$ werden in $23 \mathrm{ml}$ 2-Propanol und $3.8 \mathrm{ml}$ Wasser gelöst und nach vorsichtiger Zugabe von $0.48 \mathrm{~g}(12.6 \mathrm{mmol}) \mathrm{NaBH}_{4}$ $2 \mathrm{~h}$ bei Raumtemp. gerührt. Nach Zutropfen von $2.6 \mathrm{ml}$ Essigsäure wird $3 \mathrm{~h}$ bei $80^{\circ} \mathrm{C}$ gerührt, dann wird das Lösungsmittel i. Vak. abdestilliert, der Rückstand mit $15 \mathrm{ml} 6 \mathrm{~N} \mathrm{HCl}$ versetzt, die wäßrige Lösung zweimal mit jeweils $100 \mathrm{ml}$ EE ausgeschüttelt, mit verd. $\mathrm{NaOH}$ alkalisch gemacht und das freigesetzte Amin dreimal mit jeweils $100 \mathrm{ml} \mathrm{EE}$ ausgeschüttelt. Die vereinigten organischen Phasen werden mit Magnesiumsulfat getrocknet, das Lösungsmittel wird abdestilliert und der ölige Rückstand bei $90^{\circ} \mathrm{C} / 15$ Torr destilliert (Kugelrohrdestillation); Ausb. $0.10 \mathrm{~g}(29 \%),[\alpha]_{\mathrm{D}}^{20}=-12.9$ $(c=1.3, \mathrm{EtOH}),(S):(R)=68.2: 31.8$ (berechnet aus $[\alpha]_{\mathrm{D}}^{20}=$ $+35.4(c=1, \mathrm{EtOH})$ für reines $(R)-18^{36)}>$. e.e. der Gesamtreaktion $(R)-14 \rightarrow(S / R)-18: 38.3 \%$ [berechnet nach $\mathrm{Lit}^{37)}$ aus e.e. von eingesetztem $(R)-14: 95 \%]$

$(R / S)$-18: Wie vorstehend beschrieben aus $0.615 \mathrm{~g}(2.13 \mathrm{mmol})$ $(R / S)-17\left([\alpha]_{0}^{20}=+38.7\right), 21 \mathrm{ml}$ Propanol, $3.5 \mathrm{ml}$ Wasser, $0.438 \mathrm{~g}$ (11.6 mmol) $\mathrm{NaBH}_{4}$ und $2.3 \mathrm{ml}$ Essigsäure; Ausb. $0.08 \mathrm{~g}(28 \%)$, $[\alpha]_{\mathrm{D}}^{20}=+13.1(c=1, \mathrm{EtOH}),(R):(S)=68.5: 31.5$ (berechnet aus $[\alpha]_{\mathrm{D}}^{20}=+35.4(c=1$, EtOH $)$ für reines $\left.(R)-18^{36)}\right\rangle$. e.e. der Ge- 
samtreaktion $(S)-14 \rightarrow(R / S)-18: 38.8 \%$ [berechnet nach Lit. $^{37)}$ aus e.e. von eingesetztem $(S)-14: 95.3 \%]$.

\section{CAS-Registry-Nummern}

1a: 80-68-2 / 1 b: 632-20-2 / 1 c: 72-19-5 / 2a: 87068-79-9 / 2b: 103239-32-3 / 2c: $29588-90-7$ / 3a: $39739-08-7$ / 3b: $112458-60-3$; 3c: $106819-63-0$ / 3d: 112458-61-4 / 3e: 112458-62-5 / 3f : 11245863-6 / 4a: 112458-64-7 / 4b: 106763-26-2/4c: 106763-25-1 / 4e: 106763-28-4 / 4f: 106763-27-3 / 6b: 106780-26-1/6c: 10676331-9 / 6d: 112458-65-8 / 6'd: 112424-20-1 / 6"d: 112424-21-2 / 6e: 106763-30-8 / 6f: 106763-29-5 / 6'f: 112458-66-9/6"f: $112458-$ 67-0 / 7: 25488-25-9 / 8: 25488-28-2 / 9: 112424-22-3 / 10: 11242423-4 / (R)-11: 3976-69-0 / (S)-11 : 53562-86-0/(R)-12: 112424-24-5/ (S)-12: 112424-25-6 / 13: 112458-68-1/(R)-14: 2799-16-8/(S)-14: 2799-17-9 / (R)-15: 112424-26-7 / $(S)-15$ : 109849-51-6/(R)-16: 112424-27-8 / (S)-16: 112424-28-9 / $(R)-17: 112424-29-0 /(S)$ 17: $112424-30-3 /(R)-18:$ 28163-64-6/(S)-18: 17596-79-1 $N$-(Ethoxycarbonyl)phthalimid: 22509-74-6 / Benzol: 11-43-2 / Mesitylen: 108-67-8 / Hydrochinon-dimethylether: 150-78-7 / $O$-Methylsulfonyl- $N$-phthaloyl-(2R,3S)-threonin-methylester: 112424 31-4 / $O$-Methylsulfonyl- $N$-phthaloyl-(2S,3R)-threonin-methylester: $112424-32-5$ / $\beta$-Chlor- $\beta$-methyl- $N$-phthaloyl- $(2 S, 3 R)$-alaninmethylester: 112424-33-6

1) Zur elektrophilen Aromatensubstitution, 35. Mitteilung. - 34 . Mitteilung: F. Effenberger, D. Steegmüller, V. Null, Th. Ziegler, Chem. Ber. 121 (1988) 125.

2) F. Effenberger, Th. Weber, Angew. Chem. 99 (1987) 146; Angew. Chem. Int. Ed. Engl. 26 (1987) 142.

3) Th. Weber, Dissertation, Univ. Stuttgart, 1986.

4) 4a) Y. Izumi, I. Chibata, T. Itho, Angew. Chem. 90 (1978) 187; Angew. Chem. Int. Ed. Engl. 17 (1978) 176. - ${ }^{4 b}$ I. Wagner, H. Musso, Angew. Chem. 95 (1983) 827; Angew. Chem. Int. Ed. Engl. 22 (1983) 816. - ${ }^{4 c}$ B. Hoppe, J. Martens, Chem. Unserer Zeit 17 (1983) 41. - ${ }^{4 d)}$ M. S. Sadovnikova, V. M. Belikov, Russ, Chem. Rev. 47 (1978) 199.

5) sa) F. Effenberger, U. Burkard, J. Willfahrt, Angew. Chem 95 (1983) 50; Angew. Chem. Int. Ed. Engl. 22 (1983) 65, sowic Liebigs Ann. Chem. 1986, 314. - ${ }^{56)}$ F. Effenberger, U. Burkard, Liebigs Ann. Chem. 1986, 334.

6) 6a) O. Piccolo, F. Spreafico, G. Visentin, E. Valoti, J. Org. Chem. 50 (1985) 3945. - ${ }^{6 b)}$ Daicel Chemical Industries, Ltd. (K. Sato, Y. Murai, Inv.), Jpn. Kokai Tokkyo Koho JP 61,210,049, 18. Sept. 1986 [Chem. Abstr. 106 (1987) 66922 r]. - ${ }^{6 c)}$ J. P. Rieu, A. Bourcherle, H. Cousse, G. Mouzin, Tetrahedron 42 (1986) 4095 .

7) 7a) T. Gramstad, R. N. Haszeldine, J. Chem. Soc. 1957, 4069 . -

${ }^{7 b)}$ P. J. Stang, M. Hanack, L. R. Subramanian, Synthesis 1982 , 85. - ${ }^{7 c)}$ R. D. Howells, J. C. McCown, Chem. Rev. 77 (1977) 69.

${ }^{8)}$ C. D. Beard, K. Baum, V. Grakauskas, J. Org. Chem. 38 (1973) 3673.

9) F. Effenberger, D. Steegmüller, Chem. Ber. 121 (1988) 117.
10) R. R. Fraser, M. A. Petit, I. K. Saunders, Chem. Commun. 1971, 1450.

${ }^{11)}$ E. Fischer, W. Schmitz, Ber. Dtsch. Chem. Ges. 39 (1906) 351, 2208.

12) J. M. Waisvisz, M. G. van der Hoeven, J. van Peppen, W. C. M. Zwennis, J. Am. Chem. Soc. 79 (1957) 4520.

13) 13a) Y. Kataoka, Y. Seto, M. Yamamoto, T. Yamada, S. Kuwata, H. Watanabe, Bull. Chem. Soc. Jpn. 49 (1976) 1081. - ${ }^{13 b)}$ M. Kawai, U. Nagai, A. Tanaka, Bull. Chem. Soc. Jpn. 55 (1982) 1213.

14) 142 R. N. Haszeldine, J. M. Kidd, J. Chem. Soc. 1954, 4228. 14b) B. L. Booth, T. A. El-Fekky, J. Chem. Soc., Perkin Trans. 1, 1979, 2441.

${ }^{15)}$ H. B. Kagan, Spectroscopic Methods, Bd. 1, S. 68, Thieme, Stuttgart 1977.

16) ${ }^{6 a)}$ H. Arold, M. Eule, S. Reißmann, Z. Chem. 9 (1969) 447. ${ }_{16 b)}$ G. I. Tsuchihashi, S. Mitamura, K. Ogura, Bull. Chem. Soc. Jpn. 52 (1979) 2167.

i7) Wir danken Herrn Prof. Dr. J. J. Stezowski herzlich für die Hilfe bei der Röntgenstrukturanalyse.

18) Bestimmt wurde die Konfiguration am $\mathrm{C}-3$ relativ zu $\mathrm{C}-2$, unveröffentlichte Versuche von J. J. Stezowski.

19) G. A. Olah, Y. K. Mo, J. Org. Chem. 38 (1973) 353.

${ }^{20)}$ H. J. Bakoss, R. M. G. Roberts, A. R. Sadri, J. Org. Chem. 47 (1982) 4053.

21) R. L. Hinman, J. Lang, J. Am. Chem. Soc. 86 (1964) 3796.

22) 22a) C. C. Price, M. Lund, J. Am. Chem. Soc. 62 (1940) 310. 22b) A. Streitwieser jr., P. Stang, J. Am. Chem. Soc. 87 (1965) 4953.

23) 23a) T. Nakajima, S. Suga, T. Sugita, K. Ichikawa, Bull. Chem. Soc. Jpn. 40 (1967) 2980. - ${ }^{236)}$ T. Nakajima, S. Masuda, S. Nakashima, T. Kondo, Y. Nakamoto, S. Suga, Bull. Chem. Soc. Jpn. 52 (1979) 2377

${ }^{24)}$ M. Shinji, T. Nakajima, S. Suga, Bull. Chem. Soc. Jpn. 56 (1983) 1089.

25) J. O. Osby, M. G. Martin, B. Ganem, Tetrahedron. Lett. 1984, 2093.

${ }^{26)}$ B. Glatz, Dissertation, Universität Stuttgart, 1976.

${ }^{27)}$ G. H. L. Nefkens, G. I. Tesser, R. J. F. Nivard, Recl. Trav. Chim. Pays-Bas 79 (1960) 688.

28) K. Vogler, P. Lanz, Helv. Chim. Acta 42 (1959) 209, 211.

${ }^{29)}$ J. C. Sheehan, M. Goodman, G. P. Hess, J. Am. Chem. Soc. 78 (1956) 1365

30) M. Brenner, W. Huber, Helv. Chim. Acta 36 (1953) 1109.

${ }^{31)}$ S. Clarke, R. C. Hider, D. I. John, J. Chem. Soc., Perkin Trans I $1973,230$.

32) M. Hesse, H. Meier, B. Zeeh, Spektroskopische Methoden in der organischen Chemie, S. 133, Thieme, Stuttgart 1984.

33) A. Srinivasan, K. P. Richards, R. K. Olsen, Tetrahedron Lett. 1976, 891.

34) 34a) R. C. Cooksen, J. E. Kemp, J. Chem. Soc., Chem. Commun. 1971, 385. - ${ }^{34 b)}$ B. Gustafsson, Tetrahedron 34 (1978) 3023.

35) N. Yahiro, Chem. Lett. 1982, 1479.

36) 36a) D. Enders, H. Schubert, Angew. Chem. 96 (1984) 368; Angew. Chem. Int. Ed. Engl. 23 (1984) 365. - ${ }^{366)}$ H. Biere, C. Rufer, H. Ahrens, O. Loge, E. Schröder, J. Med. Chem. 17 (1974) 716.

${ }^{37)}$ V. Schurig, Kontakte 1985, 54.

$[240 / 87]$ 